\title{
SN 1987A after 18 Years: Mid\#Infrared Gemini and Spitzer Observations of the Remnant
}

\section{Citation}

Bouchet, Patrice, Eli Dwek, John Danziger, Richard G. Arendt, I. James M. De Buizer, Sangwook Park, Nicholas B. Suntzeff, Robert P. Kirshner, and Peter Challis. 2006. "SN 1987A after 18 Years: Mid\#Infrared Gemini and Spitzer Observations of the Remnant." The Astrophysical Journal 650 (1): 212-27. https://doi.org/10.1086/505929.

\section{Permanent link}

http://nrs.harvard.edu/urn-3:HUL.InstRepos:41399943

\section{Terms of Use}

This article was downloaded from Harvard University's DASH repository, and is made available under the terms and conditions applicable to Other Posted Material, as set forth at http:// nrs.harvard.edu/urn-3:HUL.InstRepos:dash.current.terms-of-use\#LAA

\section{Share Your Story}

The Harvard community has made this article openly available.

Please share how this access benefits you. Submit a story.

Accessibility 
The Astrophysical Journal, 650:212-227, 2006 October 10

(C) 2006. The American Astronomical Society. All rights reserved. Printed in U.S.A.

\title{
SN 1987A AFTER 18 YEARS: MID-INFRARED GEMINI AND SPITZER OBSERVATIONS OF THE REMNANT ${ }^{1}$
}

\author{
Patrice Bouchet, ${ }^{2,3}$ Eli Dwek, ${ }^{4}$ John Danziger,${ }^{5}$ Richard G. Arendt, ${ }^{6}$ I. James M. De Buizer, ${ }^{7}$ \\ Sangwook Park, ${ }^{8}$ Nicholas B. Suntzeff, ${ }^{3}$ Robert P. Kirshner, ${ }^{9}$ and Peter Challis 9 \\ Received 2006 January 18; accepted 2006 May 16
}

\begin{abstract}
Using the Gemini South $8 \mathrm{~m}$ telescope, we obtained high-resolution 11.7 and $18.3 \mu \mathrm{m}$ mid-IR images of SN 1987A on day 6526 since the explosion. All the emission arises from the equatorial ring. Nearly contemporaneous spectra obtained at 5-38 $\mu \mathrm{m}$ with the Spitzer Space Telescope show that this is thermal emission from silicate dust that condensed out in the red giant wind of the progenitor star. The dust temperature is $166_{-12}^{+18} \mathrm{~K}$, and the emitting dust mass is $2.6_{-1.4}^{+2.0} \times 10^{-6} M_{\odot}$. Comparison of the Gemini $11.7 \mu \mathrm{m}$ image with Chandra X-ray images, HST UV-optical images, and ATCA radio synchrotron images shows generally good correlation across all wavelengths. If the dust resides in the diffuse X-ray-emitting gas then it is collisionally heated. The IR emission can then be used to derive the plasma temperature and density, which were found to be in good agreement with those inferred from the X-rays. Alternatively, the dust could reside in the dense UV-optical knots and be heated by the radiative shocks that are propagating through the knots. In either case the dust-to-gas mass ratio in the CSM around the supernova is significantly lower than that in the general interstellar medium of the LMC, suggesting either a low condensation efficiency in the wind of the progenitor star or the efficient destruction of the dust by the SN blast wave. Overall, we are witnessing the interaction of the SN blast wave with its surrounding medium, creating an environment that is rapidly evolving at all wavelengths.
\end{abstract}

Subject headings: dust, extinction — infrared: ISM — supernovae: individual (SN 1987a) — supernova remnants

\section{INTRODUCTION}

Since its explosion, SN 1987A has evolved from a supernova (SN) dominated by the emission from the radioactive decay of ${ }^{56} \mathrm{Co},{ }^{57} \mathrm{Co}$, and ${ }^{44} \mathrm{Ti}$ in the ejecta to a supernova remnant whose emission is dominated by the interaction of the supernova blast wave with its surrounding medium. The medium surrounding the $\mathrm{SN}$ is dominated by the well-known circumstellar envelope (CSE), which consists of an inner equatorial ring (ER) flanked by two outer rings (Burrows et al. 1995), possibly part of an hourglass structure.

The collision between the ejecta of SN 1987A and the ER predicted to occur sometime in the interval 1995-2007 (Gaensler et al. 1997; Borkowski et al. 1997) is now underway. At UV-

\footnotetext{
${ }^{1}$ Based on observations obtained at the Gemini Observatory, which is operated by the Association of Universities for Research in Astronomy (AURA), Inc., under cooperative agreement with the National Science Foundation (NSF) on behalf of the Gemini partnership: the NSF (United States), the Particle Physics and Astronomy research Council (United Kingdom), the National Research Council (Canada), CONICYT (Chile), the Australian Research Council (Australia), CNPq (Brazil), and CONICET (Argentina).

${ }^{2}$ GEPI, Observatoire de Paris, Site de Meudon, 5 place Jules Janssen, 92195, Meudon, France; patrice.bouchet@obspm.fr.

${ }_{3}$ Cerro Tololo Inter-American Observatory, National Optical Astronomy Observatory, Casilla 603, La Serena, Chile. CTIO is operated by AURA, Inc., under cooperative agreement with the NSF.

${ }^{4}$ Observational Cosmology Laboratory, Code 665, NASA Goddard Space Flight Center, Greenbelt, MD 20771.

5 Osservatorio Astronomico di Trieste, via Tiepolo, 11, Trieste, Italy.

6 Science Systems and Applications, Inc., Code 665, NASA Goddard Space Flight Center, Greenbelt MD, 20771.

7 Gemini Observatory, Southern Operations Center, c/o AURA, Inc., Casilla 603, La Serena, Chile.

8 Department of Astronomy and Astrophysics, 525 Davey Laboratory, Pennsylvania State University, University Park, PA 16802.

9 Harvard-Smithsonian Center for Astrophysics, Mail Stop 19, 60 Garden Street, Cambridge, MA 02138.
}

optical (UVO) wavelengths, "hot spots" have appeared inside the ER (Pun et al. 1997), and their brightness varies on timescales of a few months (Lawrence et al. 2000). New hot spots continue to appear as the whole inner rim of the ER lights up. The visiblelight Hubble Space Telescope (HST) image obtained in 2004 February 20 reveals a necklace of such hot spots, nearly filling a lighted ring. Ongoing monitoring at X-rays with the Chandra $X$-Ray Observatory and at radio frequencies, shows that the evolution of the emission from the ER follows a similar pattern at all wavelengths.

There exist very few mid-IR observations of supernovae in general. Therefore, SN 1987A, the closest known supernova in 400 years, gives us an opportunity to explore the mid-IR properties of supernovae and the dust in their ejecta and surrounding medium with the help of the newest generation of large-aperture telescopes and sensitive mid-IR instrumentation, such as the Thermal Region Camera and Spectrograph (T-ReCS) on the Gemini telescope, in combination with IR data obtained from the Spitzer Space Telescope (Werner et al. 2004). The T-ReCS observations of the mid-IR emission from SNR 1987A are part of our continuous monitoring of the $\mathrm{SN}$ and its surrounding medium. The first detection and analysis of mid-IR emission at the position of the supernova have been reported in Bouchet et al. (2004, hereafter Paper I).

The origin of the mid-IR emission could be line emission from atomic species, synchrotron or free-free continua, or thermal emission from dust, which is probably the dominant source of emission. In general, there are several scenarios for the origin and the heating mechanism of the dust giving rise to the latetime mid-IR emission in Type II supernovae (Graham \& Meikle 1986; Gerardy et al. 2000). Thermal mid-IR emission could be (1) the emission from SN-condensed dust that is collisionally heated by reverse shocks traveling through the SN ejecta; (2) the emission from circumstellar/interstellar dust heated by the interaction of the expanding SN blast wave with the ambient medium; 

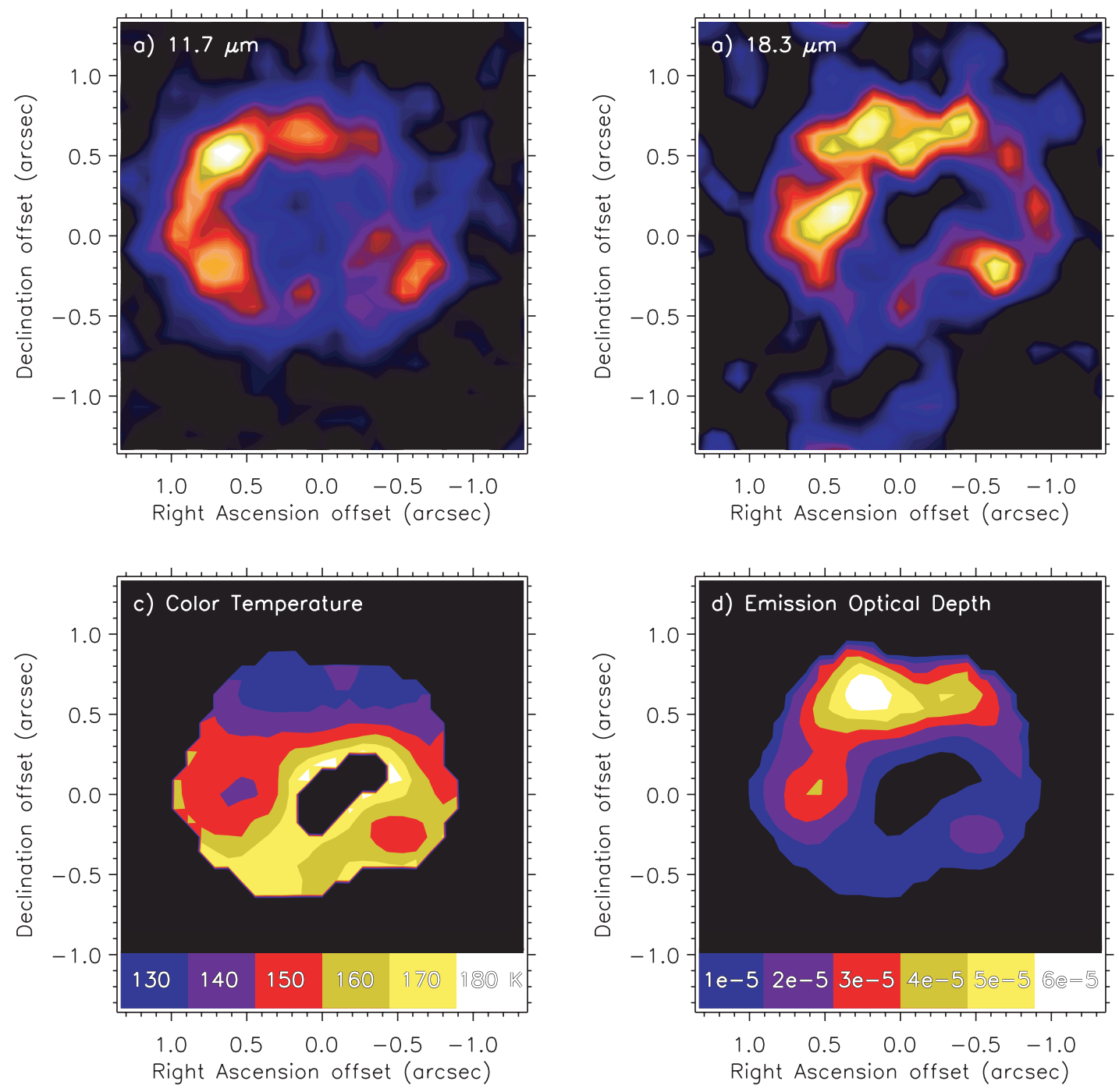

FIG. 1.-(a) SN 1987A seen with T-ReCS at day 6526 in the Si5 narrowband filter $(11.7 \mu \mathrm{m})$ and $(b)$ at day 6552 in the Qa filter $(18.3 \mu \mathrm{m})$; $(c)$ temperature map assuming pure blackbody emission and Mathis (1990) extinction law; $(d)$ opacity map resulting from the same algorithm. These images are smoothed 2 pixels $(0$ ". 18$)$.

or (3) the delayed emission (echo) from circumstellar dust radiatively heated by the early UVO supernova light curve.

Our imaging observations provide strong constraints on these possible scenarios for the mid-IR emission. They show that the bulk of the mid-IR emission is not concentrated on the center of the explosion, but arises from a ring around the SN. The morphology of the mid-IR emission can therefore be used to eliminate several scenarios for its origin. Combined with Spitzer spectroscopy, our observations can be used to determine the dust composition and temperature distribution in the ring. The Chandra $\mathrm{X}$-ray, and HST UVO data provide important constraints on the physical conditions of the medium overtaken by the supernova blast wave. These constraints can be used to determine the physical association and the heating mechanism of the dust giving rise to the mid-IR emission.

The paper is organized as follows. We first describe in $\S 2$ the imaging observations of the SN obtained by T-ReCS and compare its IR morphology to that at radio, X-ray, and UVO wavelengths. Lower resolution Spitzer mid-IR imaging observations detected SNR 1987A (including the ring) as an unresolved point source and are used in conjunction with spectroscopic data to determine the possible contribution of lines and the composition of the dust giving rise to the continuum emission. In $\S 3$ we describe the procedure used for analyzing the spectroscopic and imaging data, determining the dust composition, and presenting maps of dust temperature, IR opacities, and dust column densities. The IR image of the circumstellar medium around SN 1987A has a morphology similar to the Chandra X-ray and HST UVO images. The limited mid-IR resolution does not allow us to unambiguously determine whether the dust resides in the X-ray-emitting gas or in the UV-optical line-emitting knots in the ER. We therefore resort in $\S 4$ to an analysis of possible dust heating mechanisms and to calculating the inferred dust masses and dust-to-gas mass ratios for several possible scenarios. In $\S 5$ we discuss the evolution of the supernova and its environment as manifested from the observed light curves at various wavelengths. The results of our paper are summarized in $\S 6$.

\section{OBSERVATIONS}

\subsection{Mid-Infrared Gemini Observations}

The T-ReCS mid-IR imager/spectrometer at the Gemini $8 \mathrm{~m}$ telescope offers a combined telescope and instrument with diffraction-limited imaging $(\sim 0$."3 resolution) and superbly low thermal emissivity. On 2003 October 4 (day 6067), we imaged SNR 1987A with T-ReCS as part of the instrument's system 

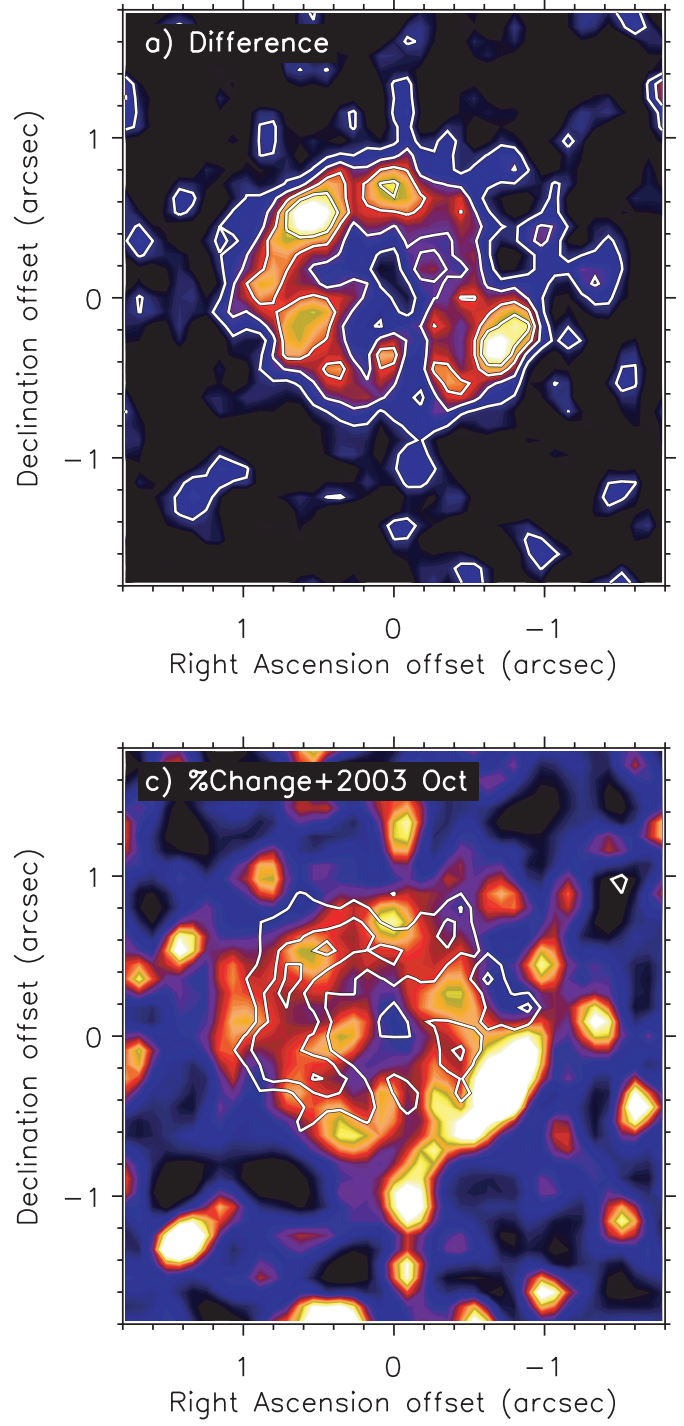
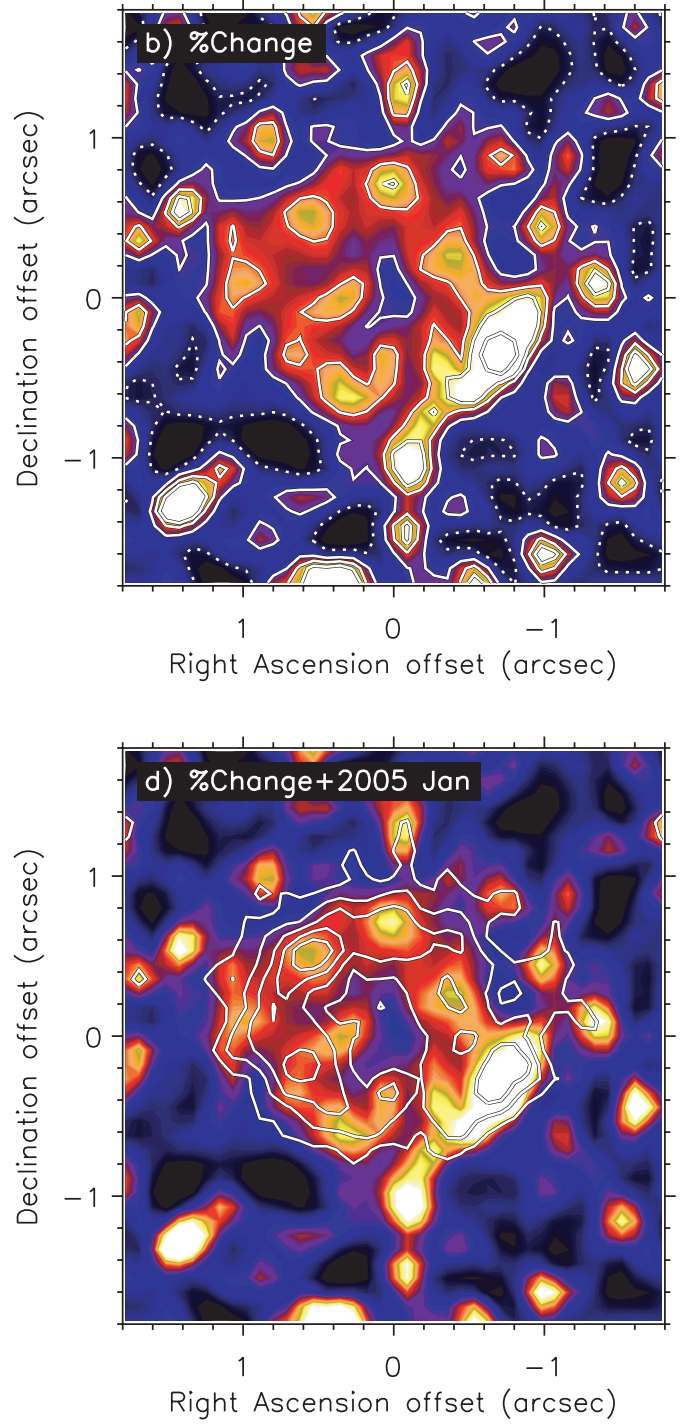

FIG. 2.-Comparison of the $N$ filter $(10 \mu \mathrm{m})$ emission at day 6067 with the Si5 filter $(11.7 \mu$ m) emission at day 6526 : $(a)$ flux-calibrated difference of the two

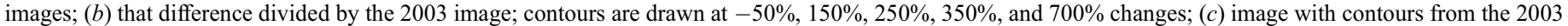
image; $(d)$ image with contours from the 2005 image. All the images are smoothed 2 pixels $(0$ ". 18).

verification program, and we reported on the detection of 10 and $20 \mu \mathrm{m}$ emission from the ER and on a $10 \mu \mathrm{m}$ emission from the supernova's ejecta (Paper I). Subsequent observations were carried out in 2005 January 6 (day 6526) in the narrow Si5 filter $\left(\lambda_{\text {eff }}=11.66 \mu \mathrm{m} ;>50 \%\right.$ transmission at $\left.\lambda=11.09-12.22 \mu \mathrm{m}\right)$ and on 2005 February 1 (day 6552) in the Qa filter $\left(\lambda_{\text {eff }}=18.30 \mu \mathrm{m}\right.$; $>50 \%$ transmission at $\lambda=17.57-19.08 \mu \mathrm{m})$. Results are presented in Figures $1 a$ and $1 b$. These images show several luminous hot spots distributed over the ring. The calibrated flux density integrated within an aperture of 1.3 radius is $F_{\nu}(11.7 \mu \mathrm{m})=$ $18.4 \pm 1.2 \mathrm{mJy}$ in the Si5 filter and $F_{\nu}(18.3 \mu \mathrm{m})=53.4 \pm 9 \mathrm{mJy}$ in the Qa filter. No color correction was applied, which would most likely have increased flux density. The standard star used for the calibration of the $11.7 \mu$ m measurement was HD 29291, whose flux density was taken to be $6.78 \mathrm{Jy}$ at $11.7 \mu \mathrm{m}$. We used $\alpha$ CMa with a flux density of $44.3 \mathrm{Jy}$ at $18.30 \mu \mathrm{m}$ for the flux calibration of the $18.3 \mu \mathrm{m}$ observation.

The blackbody color temperature corresponding to the measured fluxes at these two wavelengths is $T=185 \mathrm{~K}$ and the luminosity is $L_{\mathrm{BB}}=3.74 \times 10^{36} \mathrm{ergs} \mathrm{s}^{-1}$. We use the Mathis (1990) extinction law with $\tau_{18.3}=\tau_{11.7} / 1.35$ and $A_{18.3} / A_{J}=0.083$ and
$A_{11.7} / A_{J}=0.098$ to compute the blackbody temperature and the optical depth for each individual pixel, resulting in the maps shown in Figures $1 c$ and $1 d$. Note that in order to have the iterative algorithm converge we must assume reasonable values as a starting point. We stress that Figures $1 c$ and $1 d$ show the color temperature and optical depth maps, which are slightly different from the maps related to the physical dust as calculated in $\S 3$. They are shown only for illustrating the results obtained from our data fitted to the simplest modeling (e.g., blackbody).

In Figure 2 we compare our $11.7 \mu \mathrm{m}$ new data with the data obtained in the broad $N$ band $(10 \mu \mathrm{m})$ on 2003 October at day 6067 (Paper I). This figure shows a clear brightening in the southwest region of the ER, superimposed on a general brightening all over the ring. Given that the two images are taken with significantly different filters, we investigated whether the difference could be due to the different spectral coverage: the spectra obtained with Spitzer, which are discussed in next section, do not show any feature that could be a source of the difference. Thus, we conclude that we are observing a true brightness change.

Figures $3 a$ and $3 b$ display our images in both filters with the contours of the $0.3-8 \mathrm{keV}$ X-ray image from the Chandra X-Ray 

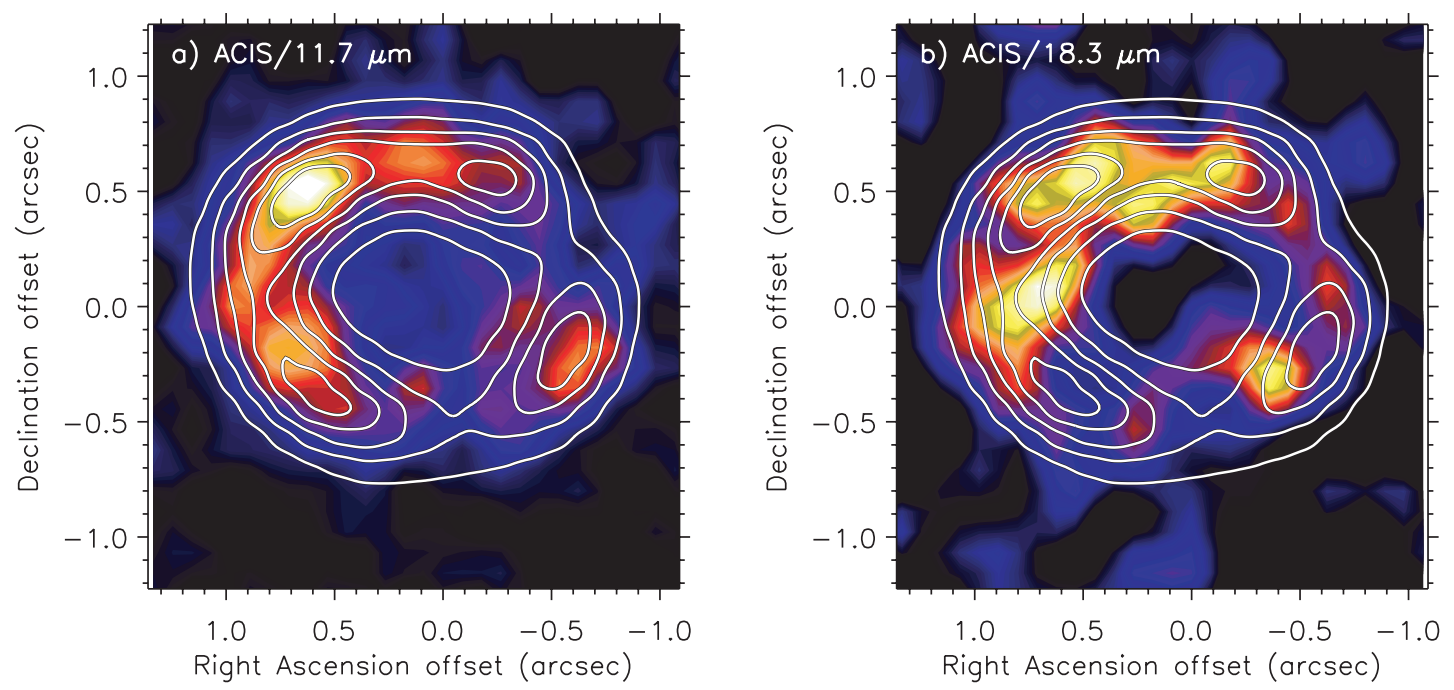

FIG. 3.- Overlays of the contour images obtained with ACIS on 2005 January 9-13 on board Chandra, superposed on the T-ReCS Si5 (a) and Qa (b) image. The T-ReCS image has been smoothed 2 pixels $\left(00^{\prime \prime} 18\right)$
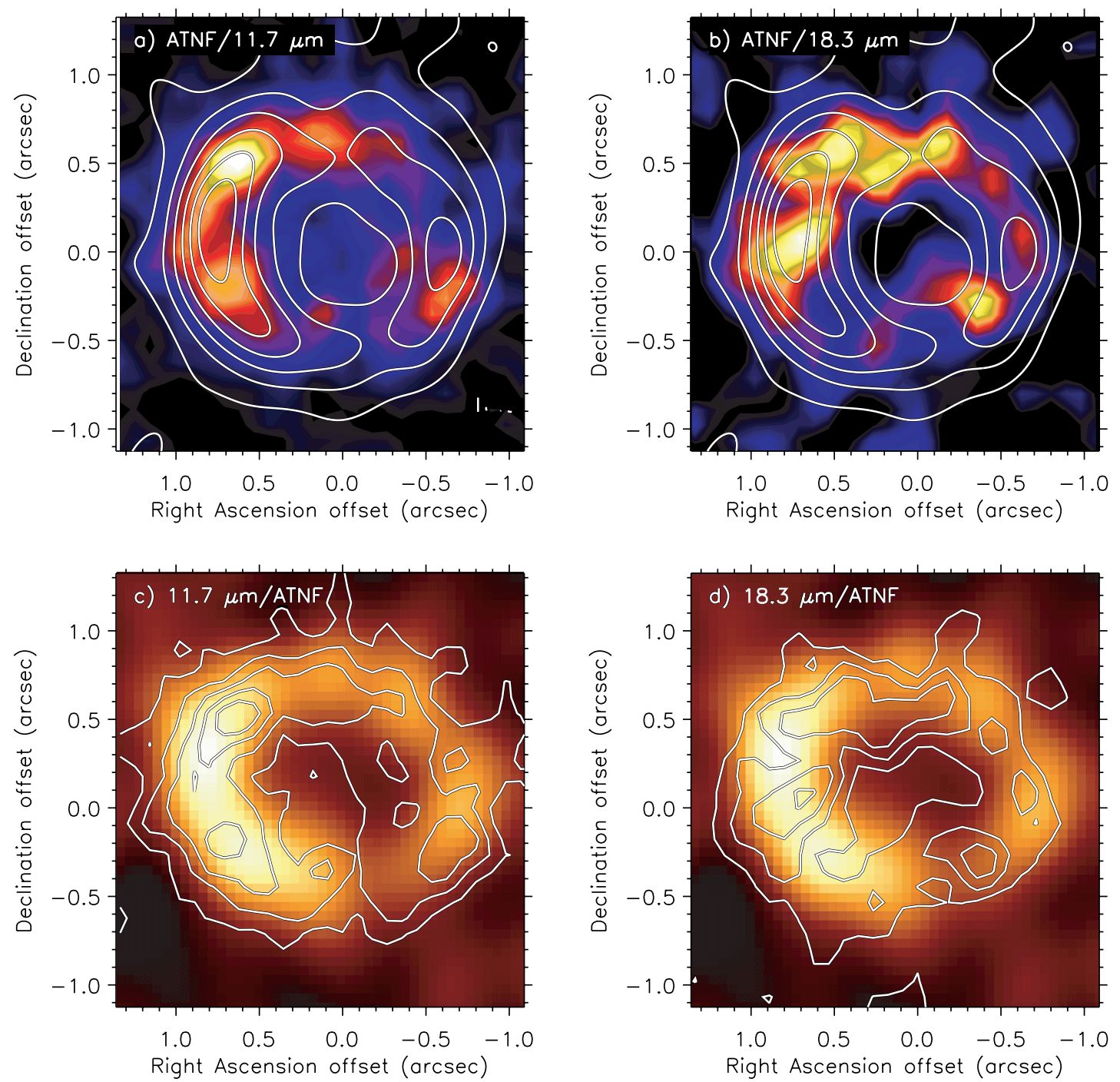

FIg. 4.-Top: Contours from the ATCA image obtained on 2003 July 31 in the $12 \mathrm{~mm}$ band (16-26 GHz) superposed on the (a) T-ReCS Si5 and (b) Qa images. Bottom: ATCA image at same frequencies obtained on 2004 May 5 (ATNF Web site) with contours from T-ReCS at (c) $11.7 \mu \mathrm{m}$ and $(d) 18.3 \mu \mathrm{m}$. The T-ReCS image have been smoothed 2 pixels $\left(00^{\prime \prime} 18\right)$. 


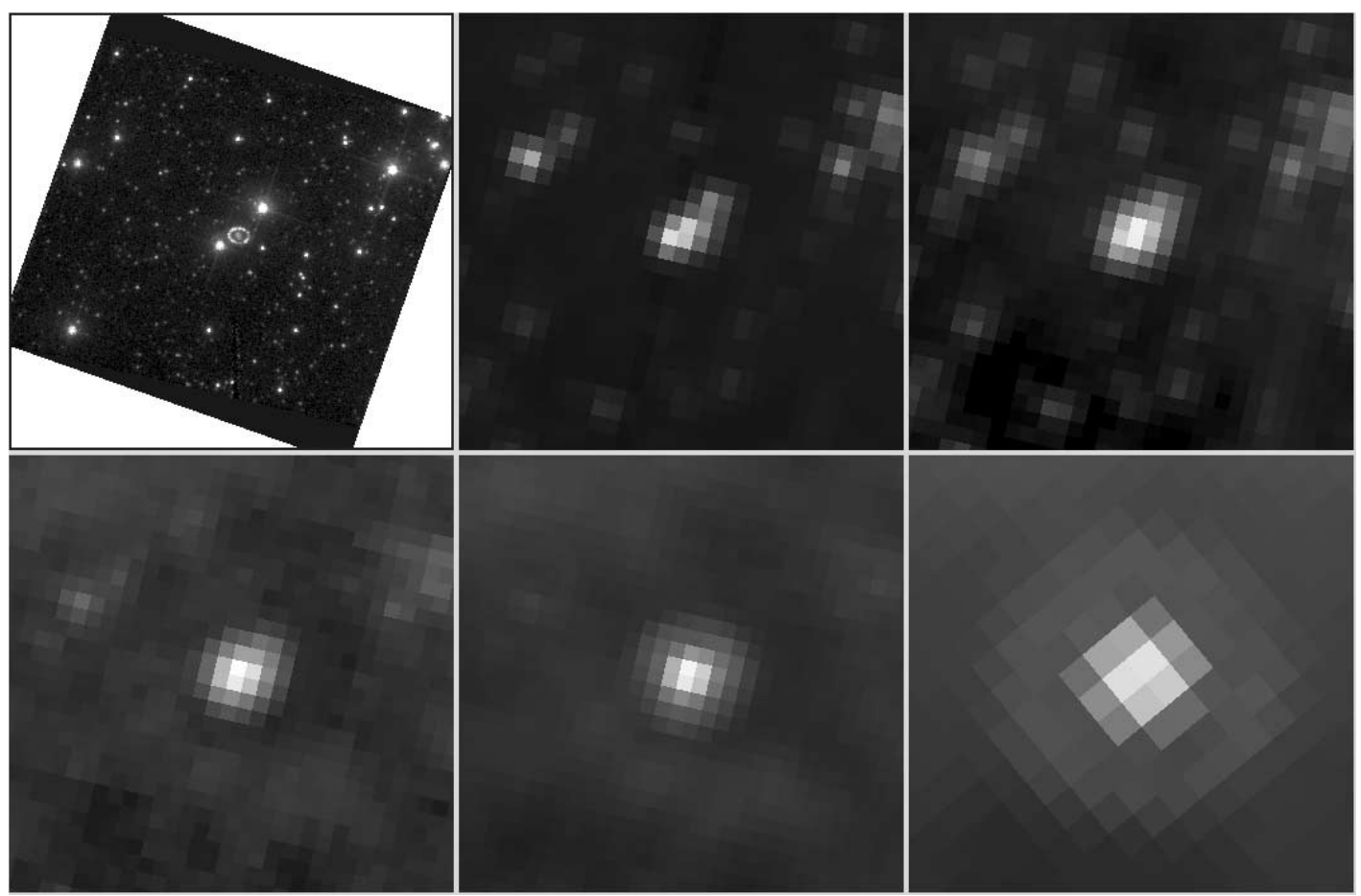

FIG. 5.-Mid-IR images of SN 1987A from Spitzer, compared to a near-IR image from HST. Left to right, top to bottom: HST ACS/HRC F814W [range $=(30,3.2 \mathrm{E} 4)$

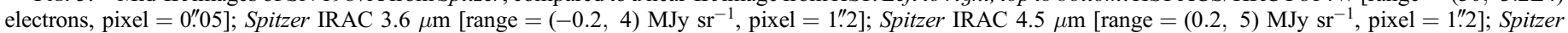
IRAC $5.8 \mu \mathrm{m}$ [range $=(1.8,8) \mathrm{MJy} \mathrm{s}^{-1}$, pixel = 1".2]; Spitzer IRAC $8 \mu \mathrm{m}$ [range $=(2,20) \mathrm{MJy} \mathrm{sr}^{-1}$, pixel $=1$.'2]; Spitzer MIPS $24 \mu \mathrm{m}$ [range $=(18,45)$ MJy sr ${ }^{-1}$, pixel = 2".45]. The bright stars in the HST image northwest and southeast of SN 1987A (star 2 and star 3) appear to be brighter than the SN emission at $3.6 \mu$ m but rapidly fade as the SN brightens at longer wavelengths. The large ring in the MIPS image is the first diffraction ring of the PSF, which is visible for any bright unresolved source. All pictures are oriented with north upward and east to the left. All span a 35".2 $\times 34$ ".6 field. The HST image is a preview image from the Multimission Archive at Space Telescope (MAST). The Spitzer images are post-BCD images from the science data archive at the Spitzer Science Center.

Observatory obtained nearly simultaneously at day $6529(2005$ January 9-13; Park et al. 2005b). The correspondence between our $11.7 \mu \mathrm{m}$ data and those obtained by Chandra is very good, although it is less so at $18.3 \mu \mathrm{m}$ because of the lower signal-tonoise ratio $(\mathrm{S} / \mathrm{N})$ in that image. A more detailed discussion of the relation between the IR and X-ray images of the ER is presented in $\S 3$ below.

Figures $4 a$ and $4 b$ show the contours of the image obtained in the $12 \mathrm{~mm}$ band (16-26 GHz) at day 6003 (2003 July 31) with the Australian Telescope Compact Array (ATCA) at the Australian National Telescope Facility (ATNF; Manchester et al. 2005) superposed on our 11.7 and $18.3 \mu \mathrm{m} \mathrm{T-ReCS}$ images, respectively. The correspondence between our $11.7 \mu \mathrm{m}$ image and the synchrotron radio emission is not as remarkable as it is with the Chandra image. Nevertheless, the $18.3 \mu \mathrm{m}$ bright spot in the east side looks better correlated with the radio lobe than X-ray spots do. Furthermore, an image obtained in 2004 May 5 (day 6298) at the same frequencies, posted on the ATNF Web site, is reproduced here in Figures $4 c$ and $4 d$. This later radio image shows better correlation with our $11.7 \mu$ m image than the radio image obtained on day 6003 , probably because it was taken closer to the epoch of the mid-IR observations. This demonstrates the importance of evolutionary effects on the morphology of the emission at all wavelengths.

There is good overall agreement in shape and size between our IR image and images obtained from the X-ray to the radio. The mean radii and approximate surface brightness distribution (brighter on the east side) of the ring are similar at all wavelengths, demonstrating that the dust is coextensive with the gas components. The origin of that brightness asymmetry may be related to an asymmetric distribution of the ejecta or the circumstellar material (CSM; Park et al. 2002, 2004) and/or to a time- dependence effect caused by the tilt of the ER, as argued by Panagia et al. (1991).

The most likely source for mid-IR radiation is thermal emission from warm dust (see discussion below). The X-ray radiation is thermal emission from very hot gas (Park et al. 2005a and references therein), whereas the optical emission arises from the dense knots in the ER that are overrun by slower shocks. The radio emission is likely to be synchrotron radiation from shockaccelerated electrons spiraling in the remnant's magnetic field, as stated by Dunne et al. (2003) and Manchester et al. (2005). Park et al. $(2002,2004)$ argue that until 2000 December, hard $(E>$ $1.2 \mathrm{keV}) \mathrm{X}$-ray and radio emissions were produced by fast shocks in the CS H II region, while the optical and $\operatorname{soft}(E<1.2 \mathrm{keV})$ $\mathrm{X}$-ray emissions came from slower shocks in the denser ER. Park et al. (2003) note that as of 2002 December 31 (day 5791), correlations between the X-ray and the optical/radio images are more complex than the above simple picture suggests, which is expected as the blast wave is reaching the main body of the inner ring.

\subsection{Spitzer Observations}

\subsubsection{Imaging Data}

Imaging of SNR 1987A was carried out with Spitzer Multiband Imaging Photometer (MIPS) at $24 \mu \mathrm{m}$ (Rieke et al. 2004) and Infrared Array Camera (IRAC) at 3.6-8 $\mu \mathrm{m}$ (Fazio et al. 2004) $($ AORIDs $=5031424$ and 5030912). Almost 1 year later SNR 1987A was again imaged with IRAC, this time serendipitously near the edge of the field of observations targeting other sources (AORIDs $=11191808$ and 11526400). All these data were obtained from the Spitzer data archive. The corresponding images are shown in Figure 5 (top left to bottom right), which 
TABLE 1

Observed FluXes From SN1987A

\begin{tabular}{|c|c|c|c|c|c|c|c|}
\hline Day $^{a}$ & Instrument & $5.8 \mu \mathrm{m}$ & $8.0 \mu \mathrm{m}$ & $10.4 \mu \mathrm{m}$ & $11.7 \mu \mathrm{m}$ & $18.3 \mu \mathrm{m}$ & $24 \mu \mathrm{m}$ \\
\hline .................... & T-ReCS & $\ldots$ & $\ldots$ & $9.9 \pm 1.5$ & $\ldots$ & $\ldots$ & $\ldots$ \\
\hline 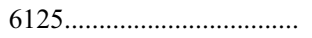 & T-ReCS & $\ldots$ & $\ldots$ & $\ldots$ & $\ldots$ & $<50.6$ & $\ldots$ \\
\hline 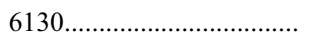 & IRAC & $1.79 \pm 0.06$ & $4.98 \pm 0.16$ & $\ldots$ & $\ldots$ & $\ldots$ & $\ldots$ \\
\hline 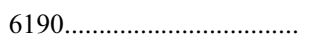 & $\mathrm{IRS}^{\mathrm{b}}$ & $\ldots$ & $\ldots$ & $\ldots$ & $13.3 \pm 0.3$ & $31.8 \pm 1.0$ & $\ldots$ \\
\hline 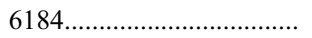 & MIPS & $\ldots$ & $\ldots$ & $\ldots$ & $\ldots$ & $\ldots$ & $29.8 \pm 1.7$ \\
\hline 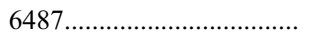 & IRAC & $2.44 \pm 0.19$ & $7.48 \pm 0.19$ & $\ldots$ & $\ldots$ & $\ldots$ & $\ldots$ \\
\hline 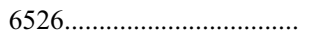 & T-ReCS & $\ldots$ & $\ldots$ & $\ldots$ & $18.4 \pm 1.2$ & $53.4 \pm 9.0$ & $\ldots$ \\
\hline
\end{tabular}

Note.-Fluxes are in units of millijanskys.

a Time elapsed since the explosion.

b Spitzer IRS data integrated between the filter half-power wavelengths.

also shows a near-IR image from HST for comparison. At 24 and $8 \mu \mathrm{m}$ SNR 1987A was detected as an unresolved point source amid a field of complex cirrus emission. At $5.8 \mu \mathrm{m}$ the source appears very slightly distorted, and at 4.5 and $3.6 \mu \mathrm{m}$ the $\mathrm{SN}$ appears to be swamped by the emission of companion stars 2 and 3 . The flux densities at 24, 8, and $5.8 \mu \mathrm{m}$ were measured using SExtractor (Bertin \& Arnouts 1996) to perform aperture photometry on the post-BCD (Basic Calibrated Data) images. Aperture radii used were 4,5 , and 6 pixels $\left(44^{\prime \prime} 8,6.0\right.$, and $\left.144^{\prime \prime} 7\right)$ at $5.8,8.0$, and $24 \mu \mathrm{m}$, respectively. Approximate aperture corrections of $1.10,1.07$, and 1.14 were applied using information from the IRAC and MIPS data handbooks. The calibrated flux densities are given in Table 1.

\subsubsection{Spectroscopic Data}

Observations of SNR 1987A were also performed with the Spitzer Infrared Spectrograph (IRS) in its short (wavelength)-low

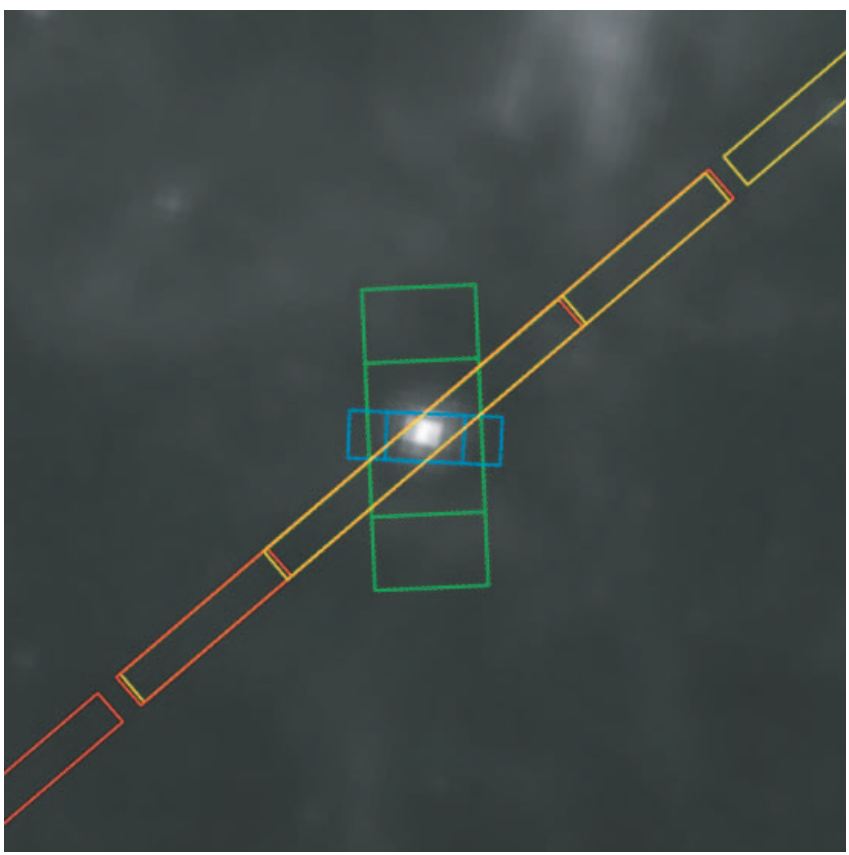

FIG. 6.-IRS slit positions superposed on the Spitzer IRAC $8 \mu \mathrm{m}$ image $\left[\right.$ range $\left.=(0,15) \mathrm{MJy} \mathrm{sr}^{-1}\right]$. This image spans an $82 !^{\prime} 8$ square field. The slit positions indicated by the long narrow red/yellow rectangles are the IRS SL first-/ second-order $(7.4-14.2 / 5.3-8.5 \mu \mathrm{m})$ pointings. The short narrow blue rectangles indicate the $\mathrm{SH}$ pointings. The short wide green rectangles indicate the $\mathrm{LH}$ pointings. For each IRS module the target is positioned at locations one-third and twothirds of the distance along the slit length. (resolution) (SL), short-high (SH), and long-high ( $\mathrm{LH})$ modes (Houck et al. 2004). These data were also obtained from the Spitzer data archive $(A O R I D=5031168)$. Figure 6 shows the slit positions for the different sets of observations. For each spectral mode we extracted SN 1987A spectra from the two-dimensional co-added post-BCD images using SPICE. ${ }^{10}$ For the SL data, the source is placed at four positions along the slit to generate spectra at two positions for two different spectral orders. For each slit position, a two-dimensional background image was generated from the median value of the images for the other three slit positions. These backgrounds were subtracted prior to extracting spectra with SPICE. For the high-resolution observations, only two slit positions were observed within a much narrower slit. So for these observations, SPICE was used to extract the SN spectrum from the columns occupied by the unresolved source, as well as a background spectrum from columns toward the opposite end of the slit. The background spectra were then subtracted from the source spectra. For all modes the spectra from the two slit positions (per spectral order) were combined using a weighted $\left(1 / \sigma^{2}\right)$ average, and (generally noisy) data where spectral orders overlapped were discarded. For the SH data, an empirically determined scaling factor of 1.38 was applied before averaging to bring results from both slit positions into agreement. An additional scale factor of 1.46 was applied to the $\mathrm{SH}$ spectrum to normalize it to

${ }^{10}$ At http://ssc.spitzer.caltech.edu/postbcd/spice.html.

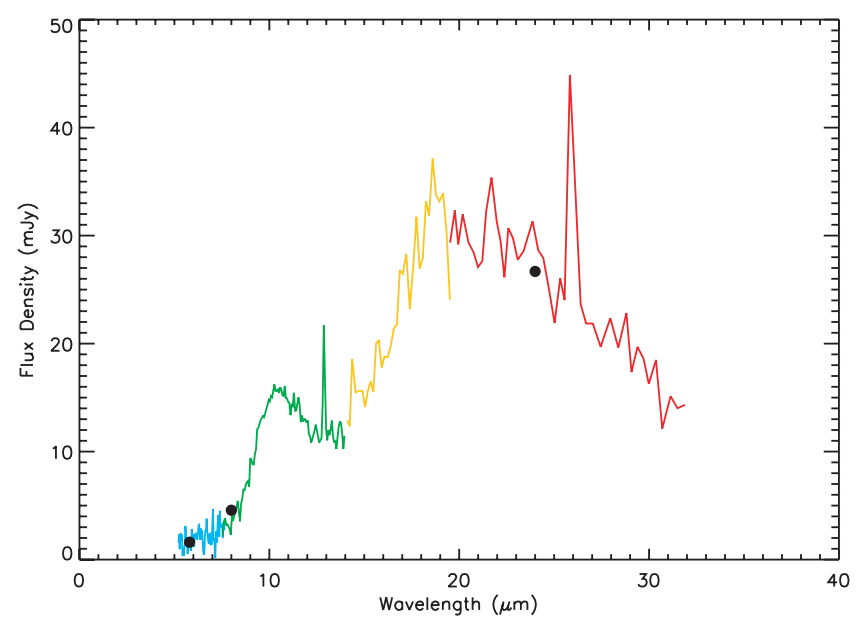

Fig. 7.-Spitzer IRS spectrum. The blue and green symbols are from the SL mode; the yellow and red are median-binned data from the SH and LH modes. The black dots are aperture photometry data points from IRAC and MIPS. 

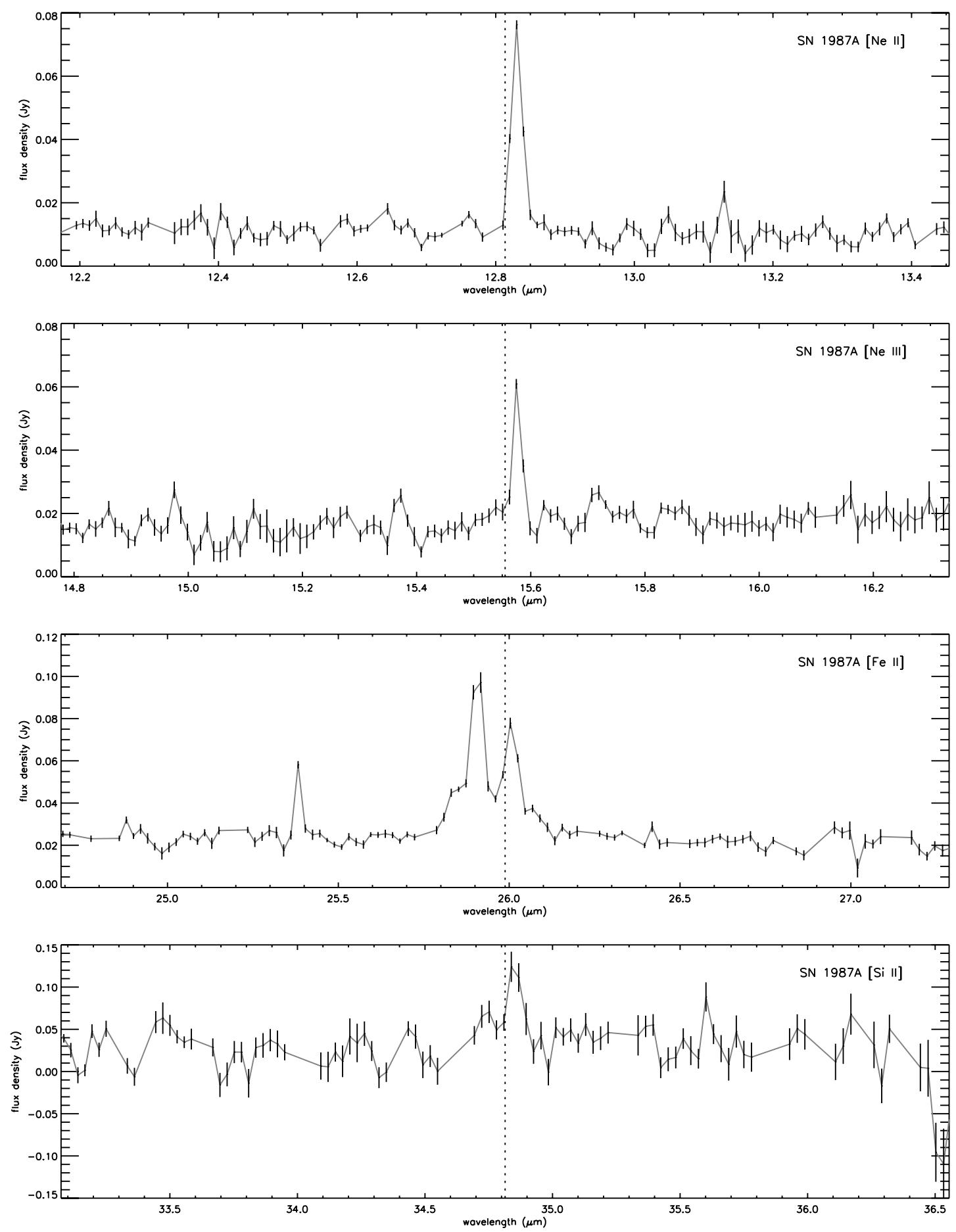

FIG. 8. - Line emission from SN 1987A observed by the IRS on Spitzer (see text).

the SL data, and a subsequent factor of 1.25 was applied to $\mathrm{LH}$ data to normalize them to the SH data. These latter scaling factors are to be expected if the subtracted background was partially contaminated by the source or in cases in which the smaller highresolution slits were slightly misaligned. Finally, for the sake of comparison with the broadband measurements and dust models, the high-resolution spectra were median-binned using intervals of 12 and 11 wavelength samples for the short and long wavelengths, respectively.

Figure 7 shows the overall calibrated spectrum, and Figure 8 displays individual profiles and identifications of the main emis- sion lines detected with the Spitzer IRS: it shows that the T-ReCS observations are dominated by the dust continuum emission, and not the lines. The [Ne II] $12.81 \mu \mathrm{m}$ and [Ne III] $15.56 \mu \mathrm{m}$ lines are, however, clearly seen. A weak [Si II] $34.81 \mu \mathrm{m}$ line remains after background subtraction, while [S III] $33.48 \mu$ m disappears entirely. Two strong lines are seen near $26 \mu \mathrm{m}$ : the redder line is [Fe II] at $25.99 \mu \mathrm{m}$ and the bluer line is [O IV] at $25.89 \mu \mathrm{m}$. Both lines could be arising from [ $\left.\mathrm{Fe}_{\mathrm{II}}\right]$ (or both from [O IV]), if they come from fast-moving ejecta on the near and far sides of the explosion, but the lack of splitting of the other lines makes this seem unlikely. A weak third component seems present here as well. 
TABLE 2

Measured IR Lines From SN1987A at Day 6190

\begin{tabular}{|c|c|c|c|c|c|}
\hline $\begin{array}{l}\text { Line Center } \\
\qquad(\mu \mathrm{m})\end{array}$ & Element & $\begin{array}{c}\text { FWHM } \\
\left(\mathrm{km} \mathrm{s}^{-1}\right)\end{array}$ & $\begin{array}{l}\text { Line Flux } \\
\left(\mathrm{W} \mathrm{cm}^{-2}\right)\end{array}$ & $\mathrm{S} / \mathrm{N}$ & Note \\
\hline $12.831 \pm 0.001 \ldots \ldots \ldots \ldots \ldots \ldots \ldots$ & {$[\mathrm{Ne} \mathrm{II}]$} & 442 & $2.392 \pm 0.185 \times 10^{-21}$ & 20 & 1 \\
\hline 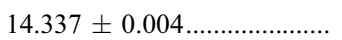 & {$[\mathrm{Ne} v]$} & 960 & $7.153 \pm 2.043 \times 10^{-22}$ & 4 & 2 \\
\hline 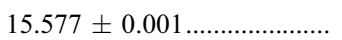 & {$[\mathrm{Ne}$ III] } & 336 & $1.038 \pm 0.141 \times 10^{-21}$ & 13 & 1 \\
\hline $24.340 \pm 0.002 \ldots \ldots \ldots \ldots \ldots \ldots \ldots$ & {$[\mathrm{Ne} v]$} & 374 & $4.819 \pm 1.265 \times 10^{-22}$ & 9 & 3 \\
\hline $25.833 \pm 0.004 \ldots \ldots \ldots \ldots \ldots \ldots \ldots$ & {$[\mathrm{F}$ IV $]$} & 472 & $4.613 \pm 1.163 \times 10^{-22}$ & 7 & 4 \\
\hline 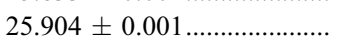 & [O IV] & 635 & $2.016 \pm 0.122 \times 10^{-21}$ & 24 & 5 \\
\hline 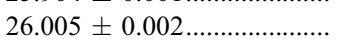 & {$[\mathrm{Fe} \mathrm{II}]$} & 844 & $1.683 \pm 0.145 \times 10^{-21}$ & 15 & 5 \\
\hline $34.850 \pm 0.008 \ldots \ldots \ldots \ldots \ldots \ldots \ldots \ldots \ldots \ldots$ & {$[\mathrm{Si}$ II] } & 603 & $1.789 \pm 0.616 \times 10^{-21}$ & 4 & 1 \\
\hline
\end{tabular}

NotEs.-(1) Line is strong in background. (2) Line was only recognized by searching for counterpart to the $24.3 \mu \mathrm{m}$ line. (3) Line is faintly visible in two-dimensional spectral data. (4) Line is not well-resolved. It could also be fit as the wing of a very broad line underneath the $[\mathrm{O} \mathrm{IV}]$ and [ $\mathrm{Fe} \mathrm{II}]$ lines. (5) Line is weak or absent in background.

If fitted as an unresolved line, this may be [F IV] at $25.83 \mu \mathrm{m}$. However, it may also be fitted as the wing of a very broad line ( $\mathrm{FWHM} \sim 2800 \mathrm{~km} \mathrm{~s}^{-1}$ ) underlying the narrower [O IV] and [ $\mathrm{Fe}$ II] lines. Such a high velocity would indicate an association with the SN ejecta rather than the ER for this line. Line fluxes, centers, and widths have been calculated by Gaussian fits using SMART (Higdon et al. 2004), and results are given in Table 2 .

\subsection{Near-IR CTIO Observations}

Near-IR $J(1.25 \mu \mathrm{m}), H(1.65 \mu \mathrm{m})$, and $K(2.2 \mu \mathrm{m})$ imaging observations of SNR 1987A were obtained on 2005 January 3-5 with the Infrared Side-Port Imager (ISPI) attached to the Blanco $4 \mathrm{~m}$ telescope at the Cerro Tololo Inter-American Observatory (CTIO). Results are displayed in Figure 9. We clearly see the ER

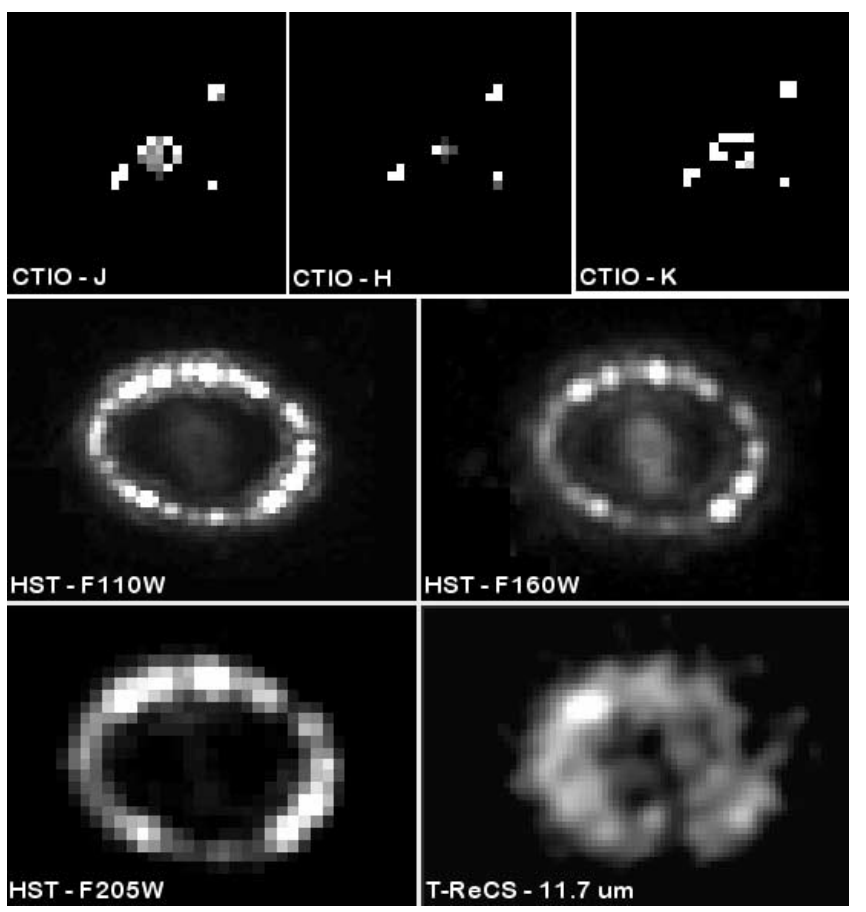

FIG. 9.-Top: SN 1987A seen with ISPI in the $J, H$, and $K$ bands with the $4 \mathrm{~m}$ Blanco telescope at CTIO; the images have been deconvolved using the multiscale maximum entropy method from Pantin \& Starck (1996). Bottom: Near-IR images obtained with HST on 2005 November 11 with the F110W $(0.8-1.4 \mu \mathrm{m})$, F160W $(1.4-1.8 \mu \mathrm{m})$, and F205W $(1.75-2.35 \mu \mathrm{m})$ filters; the T-ReCS $11.7 \mu \mathrm{m}$ image is also shown at the same scale. at $J$ and $K$, while most of the emission detected in the $H$ band arises from the supernova itself. These images have been deconvolved by using the multiscale maximum entropy method algorithm developed by Pantin \& Starck (1996). The flux densities measured in the CTIO data are $\left(F_{J}=0.33 \pm 0.25 \mathrm{mJy} ; F_{H}=\right.$ $\left.0.11 \pm 0.05 \mathrm{mJy} ; F_{K}=0.51 \pm 0.2 \mathrm{mJy}\right)$. These fluxes refer to the total of the ER+ejecta in each band (although each band seems dominated by one or the other). Images obtained with HST on 2005 November 11 through the filters F110W, F160W, and F205W are also shown in this figure, illustrating that compared to the ring, the ejecta are relatively brighter in the $H$ band (F160W) than in other bands. This clearly demonstrates the validity and the power of the deconvolution algorithm applied to our CTIO data. Note, however, that the $H S T$ bands are not exactly $J, H, K$ : one significant difference is that the Paschen $\alpha$ line is missed between the ground-based $H$ and $K$ bands, but would be seen in $H S T$ 's F205W band.

The Spitzer mid-IR spectrum indicates that dust in SN 1987A and the ER is too cool (see $\S 3$ ) to emit significantly in these nearIR bands. We believe that line emission dominates these broadband near-IR observations, and that differences in the composition, temperature, and density of the ejecta versus CSM lead to the different relative brightnesses of these structures. The young supernova remnants Cas A and Kepler may be the closest analogs we have for interpreting SN 1987A's near-IR emission. Near-IR spectra and imaging of these supernova remnants (SNRs) by Gerardy \& Fesen (2001) and Rho et al. (2003) reveal similar variation in near-IR colors. Circumstellar material (e.g., Cas A's quasistationary flocculi) is bright in $J$ due to $\mathrm{He}_{\mathrm{I}} 1.083 \mu \mathrm{m}$, and it emits strong [ $\mathrm{Fe}$ II] lines in both $J$ and $H$ bands. Weaker hydrogen lines can be detected in all bands. The ejecta in several of Cas A's fastmoving knots (FMKs) emit strong [S II] lines at $1.03 \mu \mathrm{m}$ in $J$ and weaker lines in $[\mathrm{Fe}$ II] and $\mathrm{Si}$ in various ionization states. Thus, the near-IR emission of the circumstellar ER may be dominated by $\mathrm{H}$ and $\mathrm{He} \mathrm{I}$ lines in $J$ and $H$, with synchrotron emission possibly augmenting the $K$ band (Gerardy \& Fesen 2001; Rho et al. 2003). The synchrotron processes deserve consideration in SN 1987A particularly in view of the fact, shown in Figure 4, that the radio morphology of SN 1987A reproduces the ring shape now. Moreover, the $\mathrm{H}_{2}$ molecule has many emission lines in the $K$ band and longer wavelengths. Spectra are clearly needed for testing the plausibility of the contribution from this molecule or otherwise. The first-overtone band head of molecular CO lies at $2.29 \mu \mathrm{m}$ and may be also contributing to $K$-band emission in the ring. The relatively strong $H$-band emission of SN 1987A's ejecta could reflect [Fe II] emission, because a large fraction of the ejecta should be 


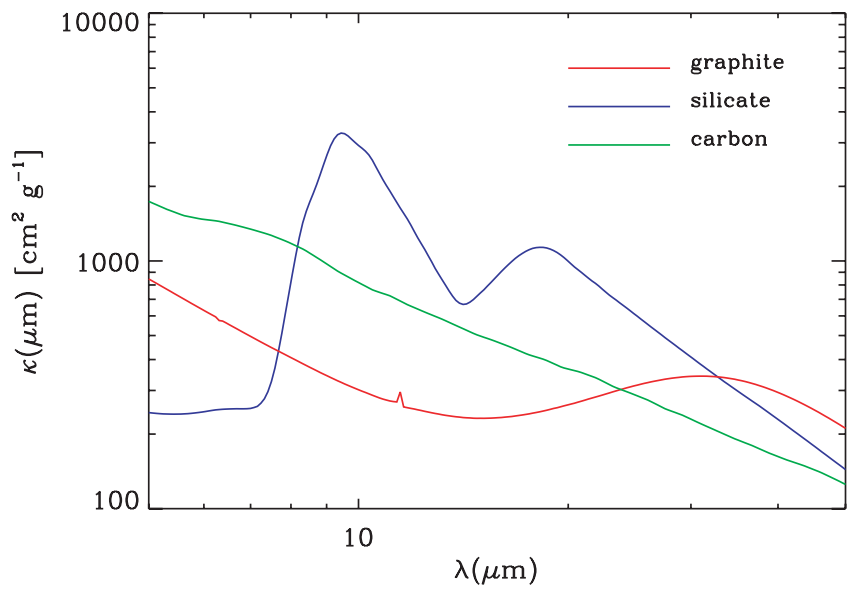

FIG. 10.- Values of the dust mass absorption coefficient, $\kappa(\lambda)$ for the three types of grains considered (references are given in the text). For small dust particles with radii $\lesssim \lambda$, the value of $\kappa(\lambda)$ is independent of grain radius.

Fe from the core of the explosion, whereas Cas A's FMKs generally consist of lighter metals originating in the outer layers of the progenitor.

\section{DATA ANALYSIS}

\subsection{The Dust Properties}

Figure 7 clearly demonstrates that the mid-IR emission is dominated by thermal emission from dust. The specific luminosity of a single dust particle of radius $a$ at temperature $T_{d}$ and wavelength $\lambda$ is given by

$$
\begin{aligned}
\ell_{\nu}(\lambda) & =4 \pi a^{2} \pi B_{\nu}\left(\lambda, T_{d}\right) Q(\lambda) \\
& =4 m_{d} \kappa(\lambda, a) \pi B_{\nu}\left(\lambda, T_{d}\right),
\end{aligned}
$$

where $B_{\nu}\left(\lambda, T_{d}\right)$ is the Planck function, $Q(\lambda)$ the dust emissivity at wavelength $\lambda$, and $\kappa(\lambda, a) \equiv 3 Q(\lambda) / 4 \rho a$ is the dust mass absorption coefficient, where $\rho$ is the mass density of the dust particle. In the Rayleigh limit, when $a<\lambda, \kappa$ is independent of particle radius. Figure 10 illustrates the $\kappa(\lambda)$ curves for amorphous carbon (Rouleau \& Martin 1991) and graphite and silicate grains (Laor \& Draine 1993) over the 5 to $30 \mu \mathrm{m}$ wavelength region. The figure shows the distinct optical properties of these dust particles over the mid-IR wavelength regime, which can greatly facilitate the identification of the emitting material with even limited broadband filters.

For an optically thin point source, the flux density, $F_{\nu}(\lambda)$, at wavelength $\lambda$ is given by

$$
F_{\nu}(\lambda)=4 M_{d} \frac{\kappa(\lambda) \pi B_{\nu}\left(\lambda, T_{d}\right)}{4 \pi D^{2}}
$$

where $M_{d}$ is the dust mass, and $D$ is the distance to the supernova, taken to be $D=51.4 \mathrm{kpc}$ (Panagia 1999).

For an extended optically thin source with an angular size $\Omega$, the surface brightness, $I_{\nu}(\lambda)$, is given by

$$
I_{\nu}(\lambda)=\Omega \tau_{d}(\lambda) B_{\nu}\left(\lambda, T_{d}\right)
$$

where $\tau_{d}(\lambda)$, the dust optical depth, is given by

$$
\tau_{d}(\lambda)=\frac{M_{d} \kappa(\lambda)}{\Omega D^{2}}
$$

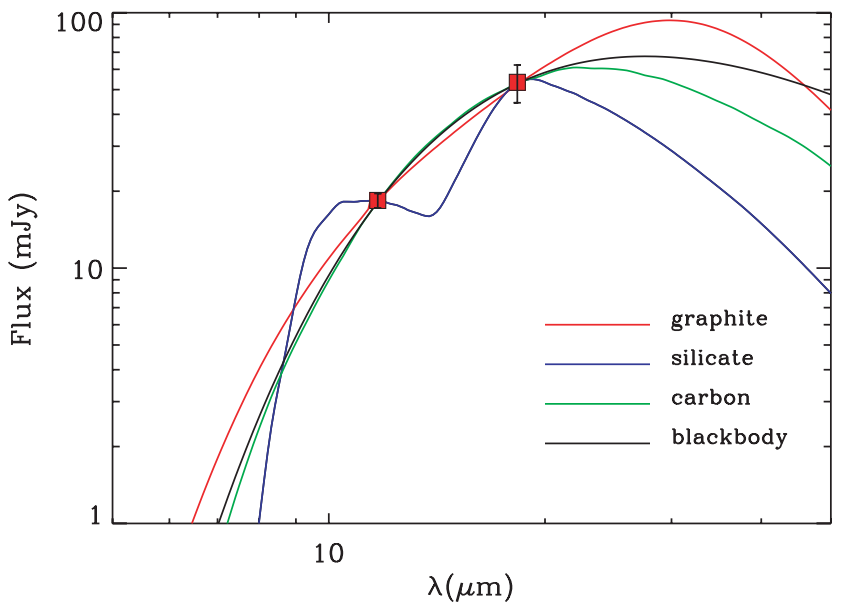

FIG. 11.-T-ReCS fluxes at 11.7 and $18.3 \mu \mathrm{m}$ fitted by our modeling of the dust continuum for three different dust compositions: silicate (blue line), carbon (green line), and graphite (red line), and in the simple blackbody case (black line). Note that the T-ReCS data alone cannot determine the dust composition.

\subsection{Spectral Analysis}

We fitted the integrated T-ReCS flux densities with a population of dust particles consisting of a single population of bare graphite, silicate, or amorphous carbon grains, using equation (2). Optical properties of silicate and graphite grains were taken from Laor \& Draine (1993), and those for amorphous carbon (BE) were taken from Rouleau \& Martin (1991). The results are given in Figure 11, which shows that the T-ReCS observations alone cannot discriminate between the different dust compositions. Comparison with the Spitzer observations clearly demonstrates that the IRS observations can be well fitted with a silicate dust composition (Fig. 12), ruling out graphite or carbon dust as major dust constituents in the CSM.

Figure 12 shows the fit of the silicate dust spectrum to the Spitzer IRS spectrum. The silicate temperature is $T_{d}=180_{-15}^{+20} \mathrm{~K}$, and the dust mass is $M_{d}=\left(1.1_{-0.5}^{+0.8}\right) \times 10^{-6} M_{\odot}$. The global parameters resulting from our model for silicates are given in

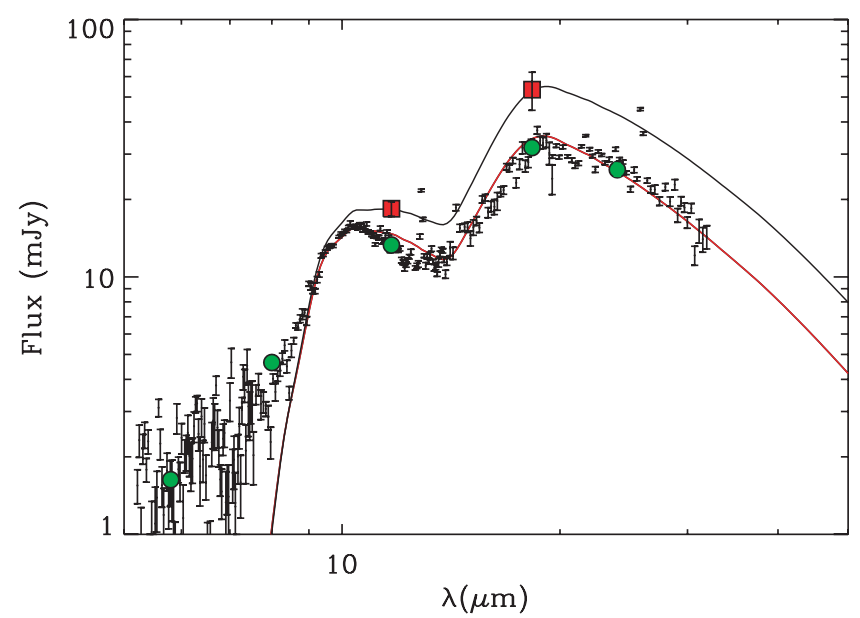

FIG. 12.-Spitzer IRS spectrum fitted with a silicate dust model. Green circles: Spitzer flux measurements. This model has been scaled to fit the T-ReCS flux measurements (red squares). Note that the mid-IR emission has been brightening significantly between both sets of observations. The Spitzer IRS data unambiguously show that silicates are the major dust component. The derived parameters of the fit are $T=180_{-20}^{+30} \mathrm{~K}, M_{\text {dust }}=(0.7-1.7) \times 10^{-6} M_{\odot}$, and $L_{\mathrm{IR}}=1.6 \pm$ $0.3 \times 10^{36} \mathrm{ergs} \mathrm{s}^{-1}$. 
TABLE 3

Global Parameters from Silicate Dust Model

\begin{tabular}{|c|c|c|c|c|}
\hline Instrument & Day $^{a}$ & $\begin{array}{l}T_{\text {dust }} \\
(\mathrm{K})\end{array}$ & $\begin{array}{c}L_{\mathrm{IR}} \\
\left(\operatorname{ergs~s}^{-1}\right)\end{array}$ & $\begin{array}{l}M_{\text {dust }} \\
\left(M_{\odot}\right)\end{array}$ \\
\hline T-ReCS ................... & 6070 & $180_{-10}^{+20}$ & $9 \pm 3 \times 10^{35}$ & $1-4 \times 10^{-6}$ \\
\hline 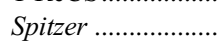 & 6190 & $180_{-15}^{+-20}$ & $1.6 \pm 0.3 \times 10^{36}$ & $0.7-1.7 \times 10^{-6}$ \\
\hline T-ReCS ........................... & 6530 & $166_{-12}^{+18}$ & $2.3 \pm 0.4 \times 10^{36}$ & $1.2-4.6 \times 10^{-6}$ \\
\hline
\end{tabular}

NoTE.-The dust mass for day 6070 has been recalculated from Paper I with the value of $\kappa$ adapted to the silicate case.

a Time elapsed since the explosion.

Table 3. Figure 13 shows the residuals of the fit, obtained by subtracting the silicate model fit from the data. The residuals are small, and their spectrum is too sharply peaked at short wavelengths to be fitted with any blackbody. The residuals are also broader than typical atomic line widths, suggesting that they may be due to solid-state features in the dust, reflecting differences in the crystalline structure of the silicates in the CSM from the average interstellar silicate dust used in the model. This figure also shows (green line) that the residuals cannot be fit by emission from amorphous carbon dust.

\subsection{Image Analysis}

Our spectral analysis showed that the mid-IR spectrum of the ER is dominated by silicate emission. We therefore used the 11.7 and $18.3 \mu \mathrm{m}$ images of the remnant to construct temperature and dust opacity maps of the circumstellar ring, using equation (3) to derive the dust temperature and equation (4) to derive the dust optical depth. In calculating these quantities, we applied a background threshold of $0.03 \mathrm{mJy}$ pixel $^{-1}$ at $11.7 \mu \mathrm{m}$ and of $0.08 \mathrm{mJy} \mathrm{pixel}^{-1}$ at $18.3 \mu \mathrm{m}$. The mass of the ring was calculated from the optical depth, over a surface area of 269 pixels, corresponding to the number of pixels that had a flux exceeding the respective thresholds at each wavelength. The average dust temperature in these maps is $T_{d}=166_{-12}^{+18} \mathrm{~K}$. The average $11.7 \mu \mathrm{m}$ optical depth per pixel is $\tau_{d}=\left(5.5_{-2.7}^{+4.2}\right) \times 10^{-6}$, and the total dust luminosity is $L_{d}=\left(2.3_{-0.4}^{+0.5}\right) \times 10^{36} \mathrm{ergs} \mathrm{s}^{-1}$, giving a dust mass $M_{d}=\left(2.6_{-1.4}^{+2.0}\right) \times 10^{-6} M_{\odot}$, in good agreement with the total mass obtained from the spectral analysis of the ER. Figure 14 shows the maps of the silicate dust temperature $(a)$ and optical depth (b) in the ER.

\section{THE ORIGIN OF THE MID-INFRARED EMISSION}

The data presented in this paper show unambiguously that the emission is thermal emission from dust. At issue are the location and heating mechanism of the dust. The forward expanding nonradiative blast wave is currently interacting with the circumstellar material and the knots in the ER. The interaction of the blast wave with the knots transmits lower velocity radiative shocks into these dense regions, producing soft X-rays and the hot spots seen in the HST images. The interaction of the blast wave with the dense knots also generates reflected shocks that propagate back into the medium that was previously shocked by the expanding SN blast wave. The complex morphology and density structure of the ER gives rise to a multitude of shocks characterized by different velocity, temperatures, and postshock densities.

The mid-IR images cannot determine the location of the radiating dust, whether it resides in the X-ray-emitting gas or in the denser UVO emitting knots. Therefore, we cannot a priori assume a particular dust-heating mechanism: collisional heating in the shocked gas or radiative heating in the radiative shocks.

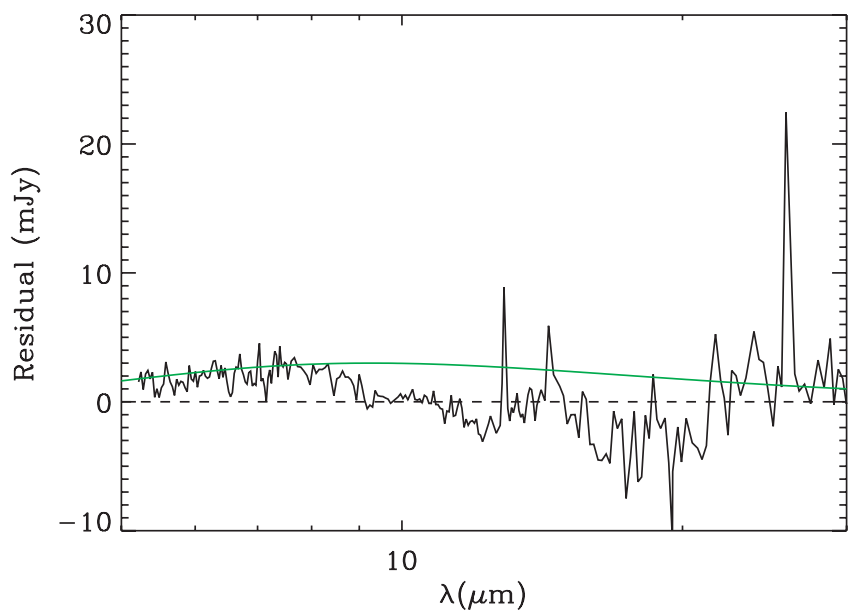

FIG. 13.- Residuals from Spitzer observations fitted with our silicate dust model. The green curve shows emission from amorphous carbon dust at $T=750 \mathrm{~K}$.

\subsection{Dust Heating Mechanism}

The relative importance of the two dust-heating mechanisms is given by the ratio (Arendt et al. 1999):

$$
\mathcal{R} \equiv \frac{H_{\mathrm{rad}}}{H_{\mathrm{coll}}}=\frac{n_{e} n_{\mathrm{H}} \Lambda\left(T_{e}\right) P_{\mathrm{abs}}}{n_{d} n_{e} \Lambda_{d}\left(T_{e}\right)},
$$

where $H_{\text {rad }}$ and $H_{\text {coll }}$ are, respectively, the radiative and collisional heating rates of the dust, $n_{e}, T_{e}$, and $\Lambda\left(T_{e}\right)$ are, respectively, the electron density, temperature, and the atomic cooling function (ergs $\mathrm{cm}^{3} \mathrm{~s}^{-1}$ ) of the gas, $P_{\text {abs }}$ is the fraction of the cooling radiation that is absorbed by the dust, and $\Lambda_{d}\left(T_{e}\right)$ is the cooling function of the gas via electronic collisions with the dust and given by

$$
\Lambda_{d}\left(T_{e}\right)=2 \bar{v}_{e} \pi a^{2} k T_{e}\langle h\rangle,
$$

where $n_{d}$ the number density of dust grains, $a$ their average radius, $\bar{v}_{e}=\left(8 k T_{e} / \pi m_{e}\right)^{1 / 2}$ is the mean thermal speed of the electrons, and $\langle h\rangle \lesssim 1$ is the collisional heating efficiency of the dust, which measures the fractional energy of the electrons that is deposited in the dust (Dwek 1987).

In the following we examine possible locations of the dust giving rise to the IR emission. For each possible site, the X-rayemitting gas or the optical knot, we determine the dominant cooling mechanism, the temperature of the dust, and the inferred dust mass. A site is viable if it can maintain a dust temperature between $\sim 150$ and $200 \mathrm{~K}$, the range of values reflecting the range of dust temperatures derived from the T-ReCS and Spitzer observations, and if the derived dust mass does not violate any reasonable abundances constraints.

\subsection{Dust in the X-Ray-Emitting Gas}

The morphological similarities between the $11.7 \mu \mathrm{m}$ emission, dust temperature, and optical depth maps, on one hand, and the X-ray maps of the supernova, on the other, suggests that the dust giving rise to the IR emission may be well mixed with the X-ray-emitting gas.

For an optically thin plasma $P_{\mathrm{abs}} \approx \tau_{d}=n_{d} \pi a^{2}\langle Q\rangle \ell$, where $\langle Q\rangle$ is the radiative absorption efficiency of the dust averaged over grain sizes and the radiation spectrum, and $\ell$ is a typical 

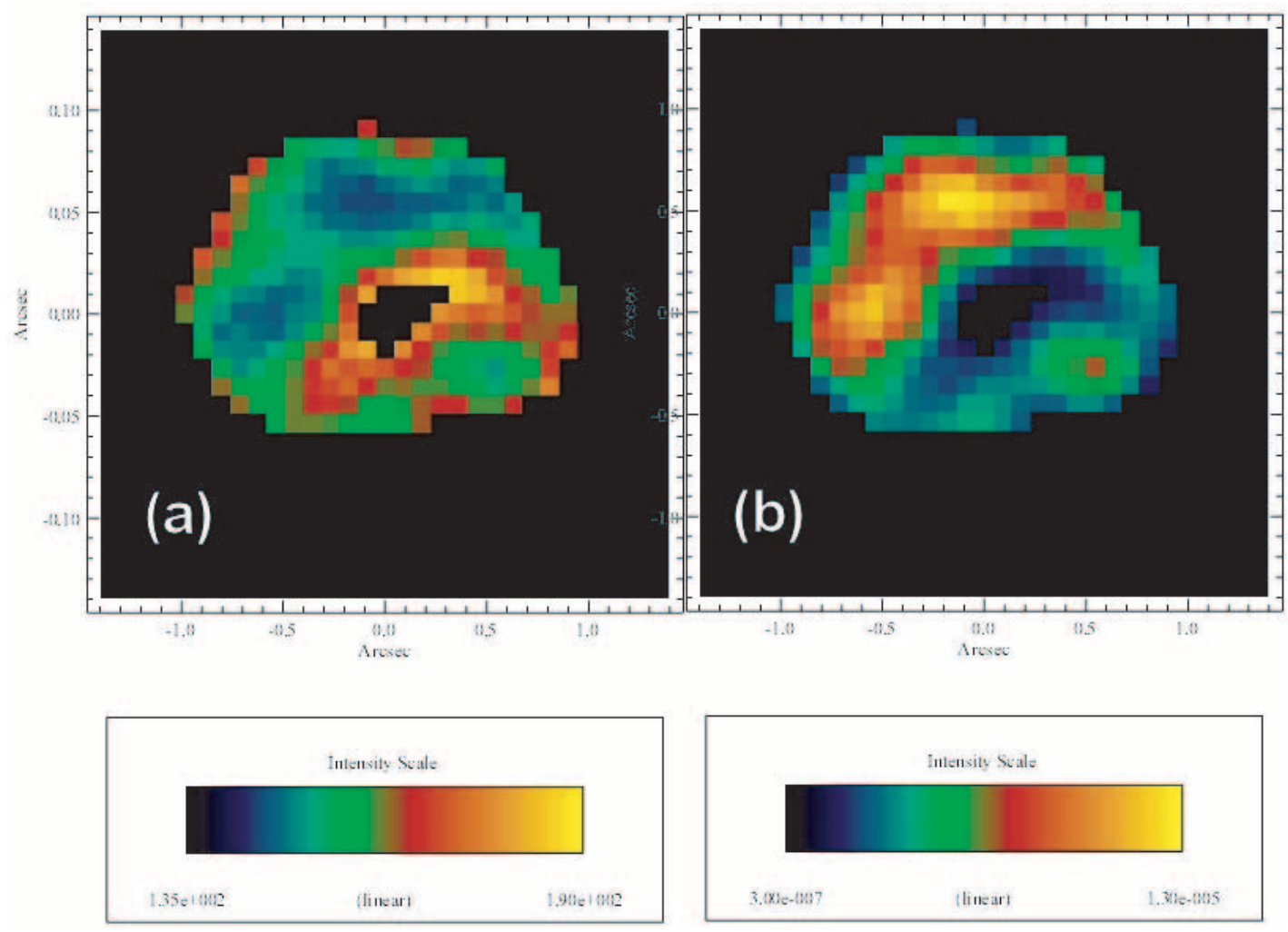

FIG. 14. - (a) Temperature and $(b)$ optical depth $\tau(11.7 \mu \mathrm{m})$ maps derived for the T-ReCS observations for a silicate dust composition.

dimension of the emitting region. Inserting this value in equation (5) we get

$$
\begin{aligned}
\mathcal{R} & =\frac{n_{e} \Lambda\left(T_{e}\right) \ell}{2 v_{e} k T_{e}} \frac{\langle Q\rangle}{\langle h\rangle} \\
& =\sqrt{\frac{\pi m_{e}}{32}} \frac{n_{e} \Lambda\left(T_{e}\right) \ell}{\left(k T_{e}\right)^{3 / 2}} \frac{\langle Q\rangle}{\langle h\rangle} .
\end{aligned}
$$

For average conditions in the X-ray plasma (see below), characterized by plasma temperatures and densities of $T_{e} \approx 10^{7} \mathrm{~K}$, and $n_{e} \approx 300-10^{3} \mathrm{~cm}^{-3}$, we get $\Lambda\left(T_{e}\right) \approx 4 \times 10^{-23} \mathrm{ergs} \mathrm{cm}^{3} \mathrm{~s}^{-1}$, and $\langle h\rangle \gtrsim 0.1$ for dust particles with radii larger than $0.05 \mu \mathrm{m}$. Adopting a value of $\langle Q\rangle \approx 1$, gives an upper limit of

$$
\mathcal{R} \lesssim 2.1 \times 10^{-20} \ell
$$

The size of the X-ray-emitting plasma is less than the radius of the ER, which is $\ell \lesssim 0.7 \mathrm{lt}-\mathrm{yr}=7 \times 10^{17} \mathrm{~cm}$, giving $\mathcal{R} \lesssim 0.14$; that is, the dust heating in the X-ray gas is dominated by electronic collisions.

\subsubsection{The Dust Temperature}

Figure 15 depicts contours of the temperature of collisionally heated silicate grains of radius $a=0.10 \mu \mathrm{m}$ as a function of plasma density and temperature. To calculate the energy deposited in the dust we used the electron ranges of Iskef et al. (1983) for energies between $20 \mathrm{eV}$ and $10 \mathrm{keV}$, and those of Tabata et al. (1972) for higher incident electron energies. We also assumed that all incident electrons penetrate the dust (no reflection). Above gas temperatures of about $3 \times 10^{6} \mathrm{~K}$, the temperature of collisionally heated dust is independent of grain radius and is very well represented by a single dust temperature, since both the radiative cooling and the collisional heating rates are proportional to the mass of the radiating dust particle. Furthermore, at these plasma temperatures the dust temperature is essentially independent of gas temperature as well and is therefore an excellent diagnostic of plasma densities. The figure shows that for plasma temperatures above $\sim 3 \times 10^{6} \mathrm{~K}$, dust temperatures between 150 and $200 \mathrm{~K}$ require electron densities of about 300 to $1400 \mathrm{~cm}^{-3}$.

X-ray observations show the presence of two X-ray emission components (Park et al. 2006): one associated with "slow shock," with an electron temperature of $\sim 0.23 \mathrm{keV}$ and a density of $n_{e} \sim$ $6000 \mathrm{~cm}^{-3}$, and the second associated with the "fast shock," with electron temperatures and densities of $k T \sim 2.2 \mathrm{keV}$ and $n_{e} \sim 280 \mathrm{~cm}^{-3}$, respectively. The latest Chandra data indicate (Park et al. 2006) that the fast shock and the slow shock of the

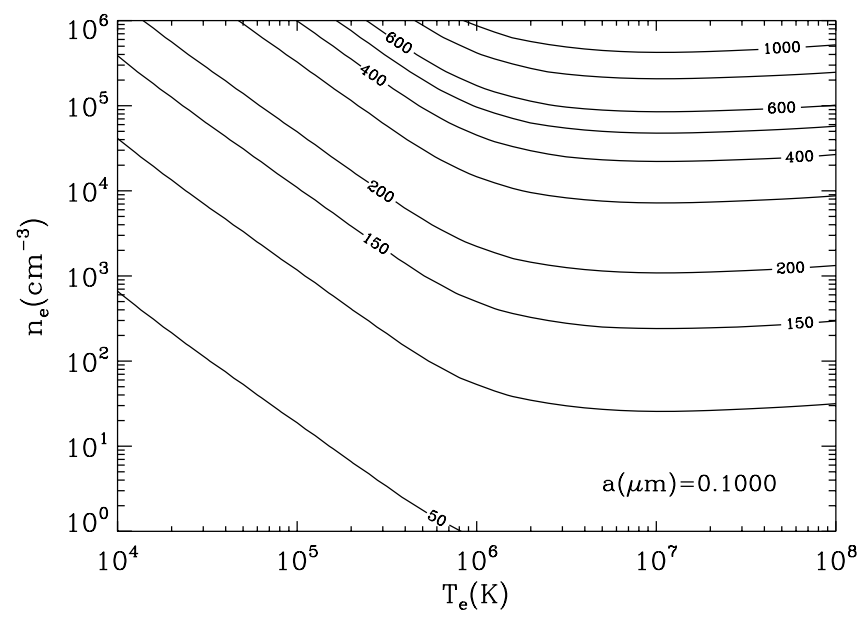

FIG. 15.-Contours of silicate dust temperature collisionally heated by a hot gas as a function of plasma temperature and electron density. 
model are becoming less distinguishable, as the overall shock front is now entering the main body of the inner ring; that is, the electron temperature of the soft component is increasing and that of the hard component is decreasing. Albeit rather speculative, the overall temperature might thus be "merging" onto an "average" temperature of $k T \sim 1.5 \mathrm{keV} \sim 1.8 \times 10^{7} \mathrm{~K}$ (Park et al. 2006) and intermediate electron densities. The range of densities expected for this average shock is in very good agreement with that implied from the IR observations.

\subsubsection{The Infrared-to-X-Ray Flux Ratio}

An important diagnostic of a dusty plasma is the infrared-toX-ray (IRX) flux ratio (Dwek 1987). For a given dust-to-gas mass ratio and grain size distribution, the IRX ratio is defined as the infrared cooling to X-ray cooling in the $0.2-4.0 \mathrm{keV}$ band and is given by

$$
\begin{aligned}
\operatorname{IRX}\left(T_{e}\right) & \equiv \frac{n_{e} n_{d} \Lambda_{d}\left(T_{e}\right)}{n_{e} n_{\mathrm{H}} \Lambda_{x}\left(T_{e}\right)} \\
& =\frac{\mu m_{\mathrm{H}} Z_{d}}{\left\langle m_{d}\right\rangle} \frac{\Lambda_{d}(T)}{\Lambda_{x}\left(T_{e}\right)},
\end{aligned}
$$

where $Z_{d} \equiv n_{d}\left\langle m_{d}\right\rangle / \mu n_{\mathrm{H}} m_{\mathrm{H}}$ is the dust-to-gas mass ratio, $\mu$ is the mean atomic weight of the gas, and $\left\langle m_{d}\right\rangle$ the mass of a dust particle, averaged over the grain size distribution. For a given value of $Z_{d}$, the IRX ratio is only a function of plasma temperature and ranges from a value of about 10 for $T_{e}=10^{6} \mathrm{~K}$ to a value of $\sim 400$ for $T_{e}=10^{8} \mathrm{~K}$. For young supernova remnants, Dwek et al. (1987a) found that the IRX ratio is significantly larger than unity for seven of the nine remnants considered in their paper, the other two having only an upper limit on their IR emission.

The observed IRX ratio can be obtained from the X-ray and IR fluxes from the SN. From the 2005 January Chandra data, we estimate the X-ray flux in the $0.2-4.0 \mathrm{keV} \mathrm{X}$-ray band to be $7.24 \times 10^{-12}$ ergs cm ${ }^{-2} \mathrm{~s}^{-1}$ after correcting for interstellar absorption by an $\mathrm{H}$ column density of $N_{\mathrm{H}}=2.35 \times 10^{21} \mathrm{~cm}^{-2}$.

Note that these values are based on the two-temperature model as used in Park et al. (2004). Fractional contributions from the soft $\left(k T_{e}=0.3 \mathrm{keV}\right)$ component in the total flux is $\sim 70 \%$ for the unabsorbed flux (and thus for $L_{\mathrm{X}}$ ). Thus, a contribution from each component in the $0.2-4 \mathrm{keV}$ X-ray flux seems to be significant, rather than it being dominated by one of them.

The total IR flux is $F_{\mathrm{IR}}=7.7 \times 10^{-12} \mathrm{ergs} \mathrm{cm}^{-2} \mathrm{~s}^{-1}$, which leads to an IRX ratio $\sim 1$. This value is lower than the values reported in Paper I, in which IRX $=6$ for the decelerated slow shock component and IRX $=3$ for the blast wave shock in the two-temperature model. It is much lower than the theoretical value of $\sim 10^{2}$, expected for a $T_{e} \approx 1.8 \times 10^{7} \mathrm{~K}$ plasma, which is observed in the young remnants Tycho and Cas A. Other (mostly older) SNRs with measurable IR emission show somewhat lower IRX ratios, but not as low as SNR 1987A.

Several effects could be the cause for this very low value of the IRX ratio in SNR 1987A. First, in remnants the IRX ratio was calculated by Dwek (1987) for an interstellar dust-to-gas mass ratio of 0.0075. The SNR 1987A blast wave is expanding into the circumstellar shell of its progenitor star, which a priori is not expected to have an interstellar dust-to-gas mass ratio. Moreover, when estimating the depletion of elements onto dust in the ER, we should compare the expected dust abundances in the ER with the maximum available abundances for the LMC. General LMC abundances exist for B stars (Rolleston et al. 1996) and the interstellar medium (ISM; Welty et al. 1999) and, although controversy remains (Korn et al. 2002), the LMC met- allicity is usually assumed to be $0.5-0.7$ solar. Assuming that the fraction of metals locked up in LMC dust is the same as in the local ISM and that the ER has the same metallicity as the LMC, then the IRX ratio in the ER should be about 0.5-0.7 times that expected from SNRs in the Milky Way, still significantly larger than implied from the observations. The extremely low value of the IRX ratio may therefore be due to a deficiency in the abundance of the dust, compared to interstellar values, which may reflect the low condensation efficiency of the dust in the circumstellar envelope.

Second, the dust deficiency could be the result of grain destruction by thermal sputtering in the hot gas. The sputtering lifetime, $\tau_{\text {sput }}$, in a plasma with temperatures above $\sim 10^{6} \mathrm{~K}$ is about (Dwek et al. 1996)

$$
\tau_{\mathrm{sput}} \approx 3 \times 10^{5} \frac{a(\mu \mathrm{m})}{n\left(\mathrm{~cm}^{-3}\right)} \mathrm{yr},
$$

where $n$ is the density of nucleons in the gas. The X-ray-emitting gas is highly ionized, and we assume that its density is that required to heat the dust to the observed range of temperatures, that is, $n \approx 300$ to $1400 \mathrm{~cm}^{-3}$. Grain destruction is important when the sputtering lifetime is about equal to the age of the shocked gas, which we take to be $\sim 1$ yr. The low IRX ratio can therefore be attributed to the effects of grain destruction if the dust particles had initial radii between 10 and $50 \AA$. So attributing the small IRX ratio to the effect of grain destruction in the hot plasma requires that only small grains had formed in the presupernova phase of the evolution of the progenitor star.

The low IRX ratio shows that IR emission from collisionally heated dust is not the dominant coolant of the shocked gas. Its lower than expected value suggests a dust-to-gas mass ratio in the ER that is only a few percent of its interstellar value. The puzzle of the low dust abundance is greater if, in fact, the IR emission arises from dust that is not embedded in the X-ray-emitting gas but from dust that resides in the UV/optical knots instead.

\subsection{The Dust-Heating Mechanism}

The possibility that the initial size of the dust grains swept up by the shock is small suggests that the temperature of the grains may not be at the equilibrium value, but may fluctuate due to the stochastic nature of the heating and cooling. Very small grains will be stochastically heated if the energy deposited in the grain in a single collision is large compared to its internal energy content and if its cooling time via IR emission is shorter than the time between subsequent collisions. Assuming that the electron and ion temperature is instantaneously equilibrated behind the shock, the mean thermal energy of the electrons will be $2.6 \mathrm{keV}$ at a postshock temperature of $2 \times 10^{7} \mathrm{~K}$. Using the electron ranges given by Iskef et al. (1983), we calculate that the energy, $\Delta E$, deposited in a dust grain of radius $a(\mu \mathrm{m})$ and density $\rho\left(\mathrm{g} \mathrm{cm}^{-3}\right)$ by electrons with energies $E \gtrsim 370 \mathrm{eV}$ is given by

$$
\Delta E(\mathrm{ergs})=5.5 \times 10^{-7} \frac{\rho a}{E(\mathrm{eV})^{0.492}},
$$

which for $\rho=3 \mathrm{~g} \mathrm{~cm}^{-3}, a=0.0050 \mu \mathrm{m}$, and $E=2.6 \mathrm{keV}$ gives an energy deposition $\Delta E=1.7 \times 10^{-10}$ ergs. This value is small compared to the internal energy of the grain, which is about $6 \times 10^{-10}$ ergs at the equilibrium grain temperature of $170 \mathrm{~K}$. The average thermal speed of the electrons is about $3 \times 10^{9} \mathrm{~cm} \mathrm{~s}^{-1}$, giving an average time between electronic collisions of about $1 \mathrm{~s}$ for an electron density of $300 \mathrm{~cm}^{-3}$ and a grain radius of 


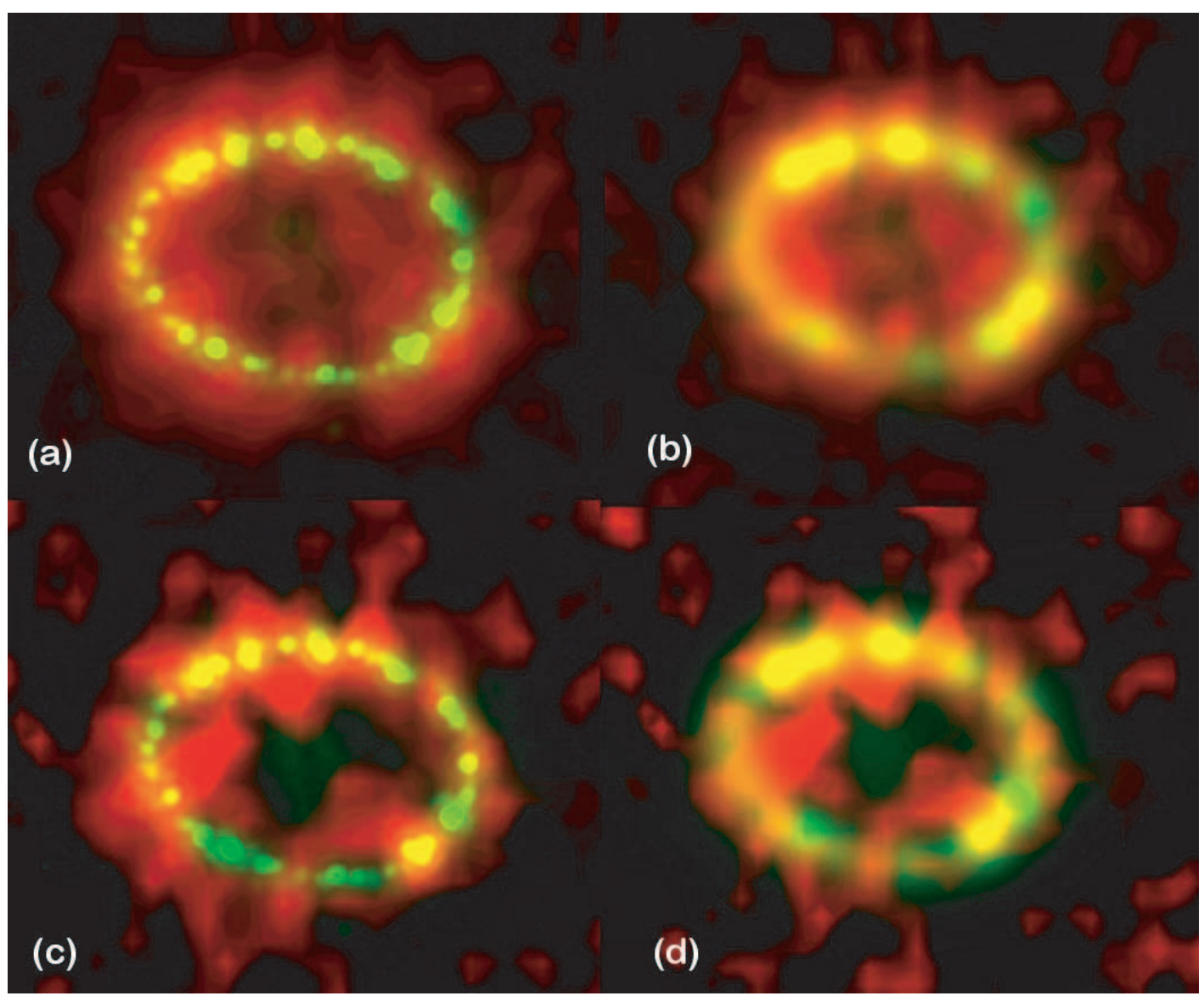

FIG. 16. - (a) Montage of the mid-IR $11.7 \mu$ m image of the ER (red) and the HST F625W image obtained on day 6502 ( yellow); (b) same as (a) with the HST image (yellow) convolved to match the resolution of the T-ReCS $11.7 \mu$ m image (red); (c) montage of the Qa image from T-ReCS (red) with the HST F625W image ( yellow); (d) same as (c) with the HST image convolved to match the T-ReCS Qa angular resolution.

$0.0050 \mu \mathrm{m}$. This is shorter than the grain's cooling time at $170 \mathrm{~K}$, which is about $10 \mathrm{~s}$ (Dwek 1986), suggesting that the dust can maintain an equilibrium temperature at that value.

Collisions with protons can deposit a significantly larger amount of energy in the dust. Typical proton energies in the shocked gas are $\sim 5 \mathrm{keV}$, and the stopping power for protons with that energy is about $240 \mathrm{MeV} \mathrm{cm}^{2} \mathrm{~g}^{-1} .{ }^{11}$ The energy deposited in a $0.0050 \mu \mathrm{m}$ radius dust particle is then about $6 \times 10^{-10} \mathrm{ergs}$, which is about equal to the internal energy of the dust grain at $170 \mathrm{~K}$. So collisions with protons and heavier nuclei can be neglected, if they are in thermal equilibrium with the electrons, because of their lower collision rate. However, if the electron and ion temperatures are not equilibrated behind the shock, then the dust heating rate will be dominated by collisions with the protons. The time between successive collisions will be about $40 \mathrm{~s}$ for a proton density of $300 \mathrm{~cm}^{-3}$. The grains should therefore cool to a temperature of about $100 \mathrm{~K}$ before another collision will take place. However, the data does not support such a broad range of dust temperatures, suggesting that the gas density could be somewhat higher (by a factor of $\sim 3$ ), in which case the dust will cool only to a temperature of $\sim 140 \mathrm{~K}$, which may be more consistent with the observations.

All these scenarios support the idea that the dust temperature in the X-ray-emitting gas does not fluctuate wildly about the equilibrium dust temperature, which can still provide strong con-

11 For NIST tabulated values, see http://physics.nist.gov/PhysRefData/Star/ Text/PSTAR.html. straints on the density and the equilibration of the electron and ion temperatures in the postshock gas.

\subsection{Dust in the Dense Knots of the Equatorial Ring}

The UVO light-emitting knots discovered with the HST resemble a string of beads uniformly distributed along the ER. Figures 16 and 17 depict the map of the dust optical depth overlaid with contour levels of the HST emission obtained on 2004 December 15 (day 6502), the closest to our $11.7 \mu \mathrm{m}$ observations. The data look very similar, but the IR emission seems to emanate from a somewhat wider region than the optical emission, an effect that cannot be entirely accounted for by the lower resolution of the IR data.

Nevertheless, the good correlation between the IR emission maps and the HST image, suggest that a significant fraction, if not most, of the mid-IR emission may be emanating from the knots. The physical conditions of a particular knot (spot 1 on the ER) have been modeled in detail by Pun et al. (2002), from the analysis of the UV/optical line emission detected by the HST Space Telescope Imaging Spectrograph (STIS). They found that the UV fluxes could be fitted with a model consisting of two shocks with velocities of $v_{s}=135$ and $250 \mathrm{~km} \mathrm{~s}^{-1}$ expanding into preshock densities of $n_{0}=3.3 \times 10^{4}$ and $10^{4} \mathrm{~cm}^{-3}$, respectively. The postshock temperatures behind the slow and fast shocks are $T_{s}=4.5 \times 10^{5}$, and $1.5 \times 10^{6} \mathrm{~K}$, with gas cooling rates of $\Lambda(T) \approx 10^{-22} \mathrm{ergs} \mathrm{cm}^{3} \mathrm{~s}^{-1}$. The cooling time of the shocked gas is given by $t_{\text {cool }}=k T_{s} / n \Lambda\left(T_{s}\right)$ and is equal to $\approx 0.1$ and $2 \mathrm{yr}$ for the slow and fast shock, respectively (Pun et al. 2002). The 


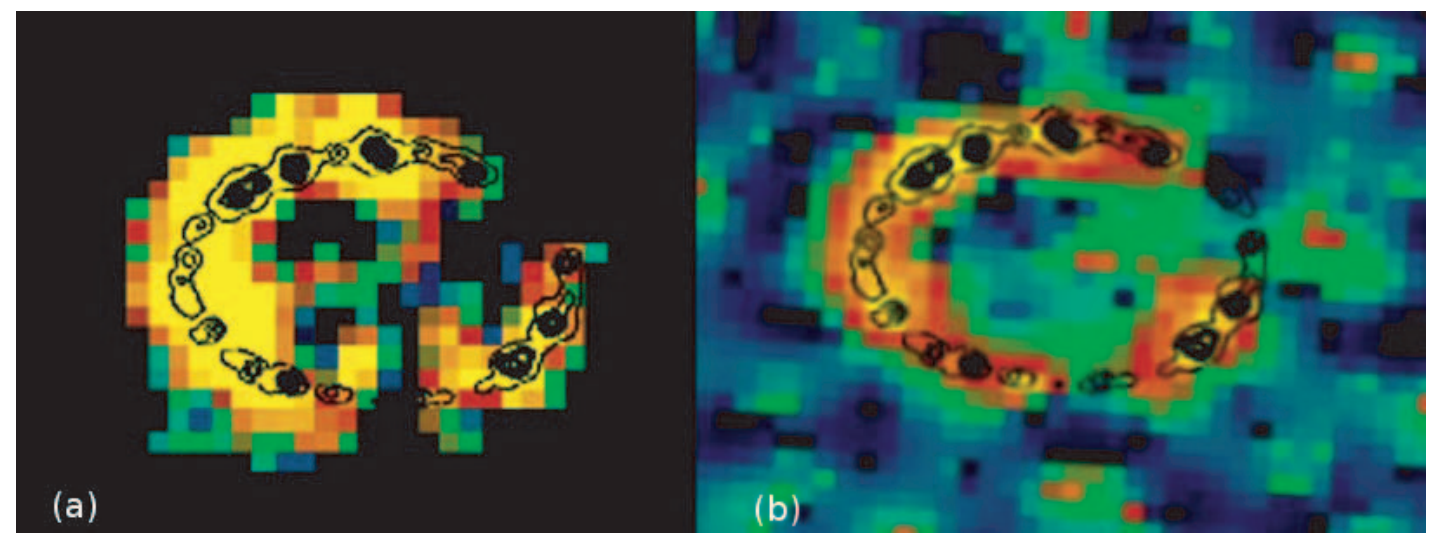

FIG. 17.- (a) Image of the ER obtained through the $11.7 \mu \mathrm{m}$ filter with T-ReCS with contours of the HST UVO image obtained on day 6502 superposed; $(b)$ same as (a) with the $11.7 \mu \mathrm{m}$ image deconvolved with the multiscale maximum entropy methodfrom Pantin \& Starck (1996).

thickness of the shock front is therefore $\sim 4 \times 10^{13}$ and $8 \times 10^{14} \mathrm{~cm}$ for the slow and fast shocks, respectively, both significantly smaller than a typical radius of the knot, which is about $2 \times 10^{16} \mathrm{~cm}$. So most of the dust in the knot resides in the unshocked gas and is heated by the radiation emitted from the cooling shocked gas.

The radiative energy density seen by the dust is approximately given by

$$
\begin{aligned}
U_{\mathrm{rad}} & \approx \frac{n^{2} \Lambda\left(T_{s}\right) \ell_{\mathrm{cool}}}{c} \\
& \approx n k T_{s} \frac{v_{s}}{c} \\
& \approx f_{c} n_{0} k T_{s} \frac{v_{s}}{c}
\end{aligned}
$$

where $\ell_{\text {cool }}=t_{\text {cool }} v_{s}$ and $f_{c}$ is the compression factor of the gas in the postshock region. For $T_{s}=10^{6} \mathrm{~K}, n_{0}=10^{4} \mathrm{~cm}^{-3}$, and a shock velocity of $200 \mathrm{~km} \mathrm{~s}^{-1}$ we calculate that

$$
U_{\mathrm{rad}} \approx 2 \times 10^{-9} f_{c} \operatorname{ergs~} \mathrm{cm}^{-3} .
$$

Pun et al. (2002) find that compression factors can be as large as $\approx 550$, giving radiation densities of $\sim 10^{-6} \mathrm{ergs} \mathrm{cm}^{-3}$ throughout the knot. The energy density of the local interstellar radiation field is about $3 \times 10^{-12} \mathrm{ergs} \mathrm{cm}^{-3}$. Silicate dust particles immersed in this field achieve equilibrium dust temperatures of about $15 \mathrm{~K}$ (Zubko et al. 2004). The energy density in the knot is therefore higher by a factor of $\sim 3 \times 10^{5}$ than that of the local interstellar radiation field, and the average dust temperature should therefore be higher by a factor of $\sim 8.3$ for a $\lambda^{-2}$ dust emissivity law $\left(T_{d} \propto U_{\mathrm{rad}}^{1 / 6}\right)$. This gives a typical dust temperature of $\sim 125 \mathrm{~K}$, in reasonable agreement with the observed average.

The total mass of radiating dust was found to be $\sim 10^{-6} M_{\odot}$. The typical mass of gas in a knot of radius $r=2 \times 10^{16} \mathrm{~cm}$ and density $n_{0}=10^{4} \mathrm{~cm}^{-3}$ is $\sim 10^{-4} M_{\odot}$. If $\mathcal{N} \approx 20$ is the number of knots in the ER, then the dust-to-gas mass ratio is $\sim 10^{-6} /\left(\mathcal{N} \times 10^{-4}\right) \approx 5 \times 10^{-4}$, or approximately a factor of 10 less than the average dust-to-gas mass ratio in the local ISM.

The low abundance of dust in the knots could be explained if the dust is efficiently destroyed in the shocked gas and if the transmitted shocks have already traversed most of the volume of the knots. Calculations presented by Jones (2004) show that about $49 \%$ of the silicate grains swept up by $200 \mathrm{~km} \mathrm{~s}^{-1}$ shocks expanding into a medium with a preshocked density of $0.25 \mathrm{~cm}^{-3}$ are destroyed. This fraction could be significantly higher for the densities encountered by the shocks traversing the knots. This scenario predicts that the IR emission from the knots was higher in the past, contrary to observed IR light curves (see below). Therefore, if the IR emission emanates from the knots, the low dust abundance must reflect the initial dust abundance in these objects.

\section{THE LIGHT CURVES}

The light curves at 10 and $20 \mu \mathrm{m}$ are shown in Figure 18: the absolute flux calibrations have been made using Cohen et al. (1992) 0 -magnitude fluxes $\left[F_{0}(10.0 \mu \mathrm{m})=35.24 \mathrm{mJy}\right.$, $F_{0}(11.7 \mu \mathrm{m})=28.57 \mathrm{mJy}$, and $F_{0}(18.30 \mu \mathrm{m})=10.25 \mathrm{mJy}$. It can be seen from Figure 18 that the flux arising from the ejecta at $10 \mu \mathrm{m}$ declines exponentially from day 2200 through day 4200 (the ISOCAM and OSCIR observations) until day 6000, at a rate

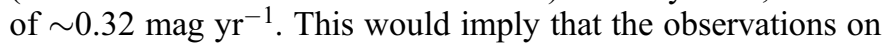

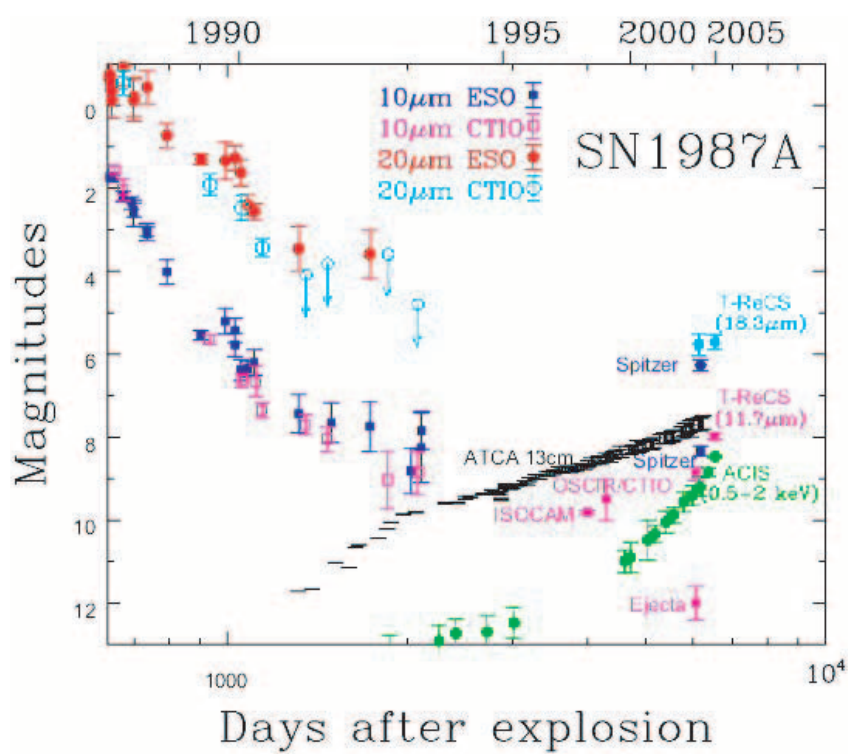

FIG. 18.- Light curves of SN 1987A for the indicated frequencies; the X-ray data points before day $\sim 4000$ are from the ROSAT data (Hasinger et al. 1996); ATCA and ROSAT ACIS fluxes are plotted in arbitrary logarithmic scales; ATCA data points are from the ATNF Web page (http://www.atnf.csiro.au/research/ SN1987A) and ACIS data are from Park et al. (2005b). The upper point in the Spitzer IRS data is integrated in the $18.3 \mu \mathrm{m}$ bandpass, and the lower point is integrated in the $11.7 \mu \mathrm{m}$ bandpass. The point labeled "Ejecta" derives from the point source near the center of the image reported in Paper I. After day 4000 all data points refer to the inner ER. 
day 4200 are not dominated by the ring emission. It is likely that the ring emission started around day 4000, at roughly the epoch when the first optical spot was discovered (Pun et al. 1997) and in good agreement with Figure 5 of Park et al. (2002), which presents ATCA and Chandra/ROSAT data. The significant increase of the fluxes reported in the present paper compared to the previous fluxes (Fig. 18) is consistent with the soft X-ray flux increase observed in the last set of data (Park et al. 2005b). This clearly shows that something has happened at around day $\sim 6000$.

This is also manifest in the radio light curve at $843 \mathrm{MHz}$ from the Molonglo Observatory Synthesis Telescope (MOST). The earlier observations by (Ball et al. 2001) from 3000 to 6000 days show a steadily increasing flux together with a steadily increasing rate of change. However, near day 6000 (R. W. Hunstead 2006 , private communication) the rate of increase undergoes a more abrupt change upward and is therefore clearly associated with the changes seen at other wavelengths. Figure 18 also shows the Spitzer data point resulting from integrating the IRS data within the appropriate bands. Including these data points shows that the trend of the increase of the flux at $11.7 \mu \mathrm{m}$ since after day $\sim 6000$ is better fitted by a "linear" function, while the increase in magnitude versus time is not linear. Although we do not draw strong conclusions, this could indicate that the shock is traveling through structures with cross sections (as seen from the center of the explosion) that do not increase with radial distance, such asshort cylindrical clouds. Sputtering timescales for all but the smallest grains are too long $(10-100 \mathrm{yr})$ to affect the IR emission, and the plasma cooling time due to the IR emission is also too long $(\sim 100 \mathrm{yr})$ to affect the time variation in the IR emission.

The $13 \mathrm{~cm}$ radio emission light curve ${ }^{12}$ is also shown in Figure 18 . No data are currently available after day 6244 , so it is premature to discuss the associated $13 \mathrm{~cm}$ evolution. It would be surprising if the rate of increase does not change upward. While the sudden increase in the X-ray light curve at day around 3700 has been interpreted as the encounter of the shock front with the first protrusions of the ER (Park et al. 2004), the "jump" after day around 6000 could be the sign of the shock reaching the main body of the ER (Park et al. 2005b). These authors also show that the light curve of the hard X-rays $(3-10 \mathrm{keV})$ is much flatter than one corresponding to the soft X-rays, similar to the radio light curves, and they argue that it is likely that the hard X-ray emission comes from the fast reverse shock rather than the decelerating forward shock, just like the radio emission (Manchester et al. 2005). We note that the reverse shock origin for the radio emission is one possible interpretation for explaining the inconsistency of the radio images with the IR and the soft X-rays images. It is not our intention to discuss evolution of radio fluxes and their relationship to other changes reported in this paper, but we note that the log-log plot in Figure 18 hides a significant change in the rate of increase of the $13 \mathrm{~cm}$ at 5000 days. The epoch of increase in the rate appears to be frequency dependent.

\subsection{The Ejecta}

An asymmetry in the profiles of optical emission lines that appeared at day 530 showed that dust had condensed in the metalrich ejecta of the supernova (Lucy et al. 1991). Although it was discovered via spectroscopy, the presence of the dust could be easily inferred from the spectral energy distribution: as the dust thermalized the energy output, after day 1000 SN 1987A radiated mainly in the mid-IR (Bouchet et al. 1991). Although the presence of the dust emission from the condensates in the ejecta was reported in Paper I, we do not detect them in the present

12 See http://www.atnf.csiro.au/research/SN1987A. observations. The possibility that this was due to the fact that the present observations were achieved with the narrow $\mathrm{Si} 5$ filter instead of the broad $N$ filter was investigated for the occurrence of the [Ne II] $12.8 \mu \mathrm{m}$ line. Indeed, this line corresponds to a fine structure transition in the ground state of $\mathrm{Ne}$ II so the temperature to excite the upper level does not need to be high if there is $\mathrm{Ne}$ II. However, Paper I reports a dust temperature of $90 \mathrm{~K}<T<$ $100 \mathrm{~K}$ in the ejecta, and there is little evidence of X-ray emission from the ejecta. Furthermore, the X-ray-emitting gas would be ionized to a much greater extent than to produce Ne II. It is thus most likely that $\mathrm{Ne}$ II is coming from warmer regions near the X-rays, which are also responsible for the other HST and Spitzer lines. Why then was the ejecta not detected in our last observations?

In Paper I the $N$-band background $\sigma$ was $0.033 \mathrm{mJy} \mathrm{pixel}^{-1}$. We used a 12 pixel area to integrate the central source, and then the $3 \sigma$ detection limit was $0.34 \mathrm{mJy}$, which is about the $0.32 \mathrm{mJy}$ measurement reported for the central source in Paper I. In the present data, although the source is observed with a better $\mathrm{S} / \mathrm{N}$, the background at $11.7 \mu \mathrm{m}$ is affected by a higher noise, with a $3 \sigma$ background value of $0.47 \mathrm{mJy}$. It transpires that we could not have detected a source at the flux level expected from the radioactive decay based light curve (e.g., $\sim 0.3 \mathrm{mJy}$ ). It appears then that the only detection of the ejecta at this late stage is achieved in the $H$ band (see Fig. 9). The origin of this emission is most likely due to line emission, although we note that the limiting flux for any continuum emitter at the center of SN 1987A, in the wavelength range $2900-9650 \AA$ A reported recently by Graves et al. (2005) (4.3 mJy in the $I$ filter) is much above our detection at $H(0.11 \mathrm{mJy})$. Therefore, our near IR results are compatible with HST observations and do not exclude contribution from a continuum.

Supernovae are known contributors to interstellar dust. The presence of isotopic anomalies in meteorites (Clayton \& Nittler 2004) and observations of Cas A (Douvion et al. 1999; Arendt, Dwek, \& Moseley 1999) and SNR 1987A (Paper I and references therein) provide direct evidence for the formation of dust in $\mathrm{SNe}$. However, the relative importance of SNe compared to quiescent outflows from AGB stars in the production of interstellar dust grains is still unclear (Jones 2004; Dwek 1998; Tielens 1998). So far, the dust masses in the ejecta have been determined observationally for two SNe, SN1987A (Lucy et al. 1989) and SN1999em (Elmhamdi et al. 2003). These masses were $\sim 3.10^{-4} M_{\odot}$ and $\sim 10^{-4} M_{\odot}$. In both cases the authors note that these values could be much higher if the dust exists in opaque clumps which may be the case. At face value however they are much less than the 0.1-1.0 $M_{\odot}$ required to make $\mathrm{SNe}$ the dominant source of interstellar dust particles. In future one must find a means of quantifying the effects of clumping. Observations of the handful of other supernovae in which an IR excess is interpreted as dust forming in the ejecta (SN 1979C and SN 1985L, and probably SN 1980K) do not allow an estimate of the mass of dust. As for the young Galactic SNR which have been observed by IRAS and ISO (Cas A, Kepler, and Tycho) the dust mass deduced is only $10^{-7}$ to $10^{-3} M_{\odot}$ (Lagage et al. 1996; Dwek et al. 1987 b), also many orders of magnitude lower than the solar mass quantities predicted.

Paper I discusses the role of supernovae in dust production. We showed there that the dust which condensed in the ejecta of SN 1987A has survived 16 years since outburst, and was still radiating the energy released by the radioactive decay of 44Ti at the expected level. Unfortunately, we could not accurately estimate the mass of the dust, and the observations reported in this paper do not allow it either, in the absence of detection of the ejecta. Thus, if we consider SN 1987A as an archetype, our data 
can neither support nor rule out the hypothesis that supernovae are significant sources for dust production.

\section{CONCLUSIONS}

We have presented mid-IR images of SNR 1987A obtained with T-ReCS on the Gemini South telescope on day 6526 at 11.7 and $18.3 \mu \mathrm{m}$ and with IRAC (5.8 and $8 \mu \mathrm{m})$ on day 6130 and MIPS $(24 \mu \mathrm{m})$ on day 6187 onboard Spitzer, together with 3$37 \mu \mathrm{m}$ spectroscopic observations of the remnant obtained with IRS on day 6190 at the same observatory. The imaging observations (Fig. 1) show that the mid-IR emission arises from the dust in the equatorial ring (ER) heated up by the interaction of the SN blast wave with its circumstellar medium. Several theoretical models predicted the presence of dust in the CSE of SN 1987A which was produced in the winds of the supergiant phase. The location of the IR emission rules out the possibility that the dust condensed out in the SN ejecta, strongly suggesting a circumstellar origin instead.

The 3-37 $\mu \mathrm{m}$ spectrum (Fig. 12) shows that the emission arises from a population of astronomical silicate particles. Temperature maps show that the dust temperature is fairly uniform in the ER and about $166_{-12}^{+18} \mathrm{~K}$, with total dust masses of $\sim 2.6_{-1.4}^{+2.0} \times 10^{-6} M_{\odot}$.

A comparison with Chandra and HST observations shows an equally good correlation between the $11.7 \mu \mathrm{m}$ IR and the X-ray and UV-optical images of the supernova. Because of the limited angular resolution in the IR we cannot determine the location or heating mechanism of the radiating dust.

The dust could be residing in the hot $\sim 10^{7} \mathrm{~K}$ gas and collisionally heated by the X-ray emitting plasma. The dust temperature is then an excellent diagnostic of the electron density, giving a value of $\sim 300-1400 \mathrm{~cm}^{-3}$, similar to the value suggested by the Chandra observations.

Comparison of the IR and X-ray fluxes suggests that the dust is depleted by a factor of $\sim 30$ in the X-ray-emitting gas, compared to its value in the local ISM of the LMC. This low value could be due to its destruction by thermal sputtering in the shocked gas or due to requiring the initial grain radii to be below $\sim 50 \AA$.

Alternatively, the dust could be residing in the UV-optical emitting knots in the ER, being radiatively heated by the cooling gas that was excited by the shocks propagating through these knots. A simple calculation for a particular knot shows that the radiative energy density can heat the dust to typical temperatures of about $125 \mathrm{~K}$, similar to those inferred from the IR observations. A comparison with the mass of the knots shows that the dust-to-gas mass ratio in the knots is lower by a factor of $\sim 10$ compared to its value in the ISM of the LMC. This low abundance reflects the low condensation efficiency of the dust in the outflow of the progenitor star.

We stress that in order to assess the role of SNe in the production of dust in the universe, it is clearly important to measure the presence of dust that survives into the formation of the remnant, and for this mid-IR and submillimeter observations are critical.

P. B. is most grateful to Eric Pantin for the use of his multiscale maximum entropy method program for the deconvolution of the images and for helpful discussions related to it. The authors acknowledge R. Manchester for providing the ATCA image obtained on 2003 July 31 . This work is based in part on observations made with the Spitzer Space Telescope, which is operated by the Jet Propulsion Laboratory, California Institute of Technology, under a contract with NASA. SMART was developed by the IRS Team at Cornell University and is available through the Spitzer Science Center at Caltech. J. D. acknowledges support by grants from MIUR COFIN, Italy. N. B. S. acknowledges support for the study of SN 1987A through the HST grants GO-8648 and GO-9114 for the Supernova Intensive Survey (SInS; PI: Robert Kirshner). S. P. was in part supported by the SAO grant GO5-6073X. E. D. was supported in part by NASA LTSA 2003.
Arendt, R. G., Dwek, E., \& Moseley, S. H. 1999, ApJ, 521, 234

Ball, L., et al. 2001, ApJ, 549, 599

Bertin, E., \& Arnouts, S. 1996, A\&AS, 117, 393

Borkowski, K. J., Blondin, J. M., \& McCray, R. 1997, ApJ, 477, 281

Bouchet, P., Danziger, I. J., \& Lucy, L. B. 1991, AJ, 102, 1135

Bouchet, P., et al. 2004, ApJ, 611, 394 (Paper I)

Burrows, C. J., et al. 1995, ApJ, 452, 680

Clayton, D. D., \& Nittler, L. R. 2004, ARA\&A, 42, 39

Cohen, M., et al. 1992, AJ, 104, 1650

Douvion, T., Lagage, P. O., \& Césarsky, C. J. 1999, A\&A, 352, L111

Dunne, L., et al. 2003, Nature, 424, 285

Dwek, E. 1986, ApJ, 302, 363

1987, ApJ, 322, 812

1998, ApJ, 501, 643

Dwek, E., Foster, S. M., \& Vancura, O. 1996, ApJ, 457, 244

Dwek, E., et al. 1987a, ApJ, 320, L27 1987b, ApJ, 315, 571

Elmhamdi, A., et al. 2003, MNRAS, 338, 939

Fazio, G., et al. 2004, ApJS, 154, 10

Gaensler, B. M., et al. 1997, ApJ, 479, 845

Gerardy, C. L., \& Fesen, R. A. 2001, AJ, 121, 2781

Gerardy, C. L., et al. 2000, AJ, 119, 2968

Graham, J. R., \& Meikle, W. P. S. 1986, MNRAS, 221, 789

Graves, G. J. M., et al. 2005, ApJ, 629, 944

Hasinger, G., Aschenbach, B., \& Truemper, J. 1996, A\&A, 312, L9

Higdon, S. J. U., et al. 2004, PASP, 116, 975

Houck, J., et al. 2004, ApJS, 154, 18

Iskef, H., Cunningham, J. W., \& Watt, D. E. 1983, Phys. Med. Biol., 28, 535

Jones, A. P. 2004, in ASP Conf. Ser. 309, Astrophysics of Dust, ed. A. N. Witt,

G. C. Clayton, \& B. T. Draine (San Francisco: ASP), 347

Korn, A. J., et al. 2002, A\&A, 385, 143

Lagage, P. O., et al. 1996, A\&A, 315, L273

\section{REFERENCES}

Laor, A., \& Draine, B. T. 1993, ApJ, 402, 441

Lawrence, S. S., et al. 2000, ApJ, 537, L123

Lucy, L. B., et al. 1989, in IAU Coll. 120, Structure and Dynamics of the ISM, ed. G. Tenorio-Tagle, M. Moles, \& J. Melnick (New York: Springer), 164 1991, in The Tenth Santa Cruz Workshop, ed. S. E. Woosley (New York: Springer), 82

Manchester, R. N., Gaensler, B. M., Staveley-Smith, L., Kesteven, M. J., \&

Tzioumis, A. K. 2005, ApJ, 628, L131

Mathis, J. S. 1990, ARA\&A, 28, 37

Panagia, N. 1999, in IAU Symp. 190, New Views of the Magellanic Clouds, ed.

Y.-H. Chu, N. Suntzeff, J. Hesser, \& D. Bohlender (San Francisco: ASP), 549

Panagia, N., et al. 1991, ApJ, 380, L23

Pantin, E., \& Starck, J.-L. 1996, A\&AS, 118, 575

Park, S., et al. 2002, ApJ, 567, 314

2003, in IAU Symp. 218, Young Neutron Stars and Their Environ-

ments, ed. B. Gaensler \& F. Camilo (San Francisco: ASP), 154

Park, S., et al. 2004, ApJ, 610, 275

- 2005a, Adv. Space Res., 35, 991

. 2005b, ApJ, 634, L73

. 2006, ApJ, 646, 1001

Pun, C. S. J., et al. 1997, IAU Circ., 6665, 1

2002, ApJ, 572, 906

Rho, J., et al. 2003, ApJ, 592, 299

Rieke, G., et al. 2004, ApJS, 154, 25

Rolleston, W. J. R., et al. 1996, A\&A, 315, 95

Rouleau, F., \& Martin, P. G. 1991, ApJ, 377, 526

Tabata, T., Ito, R., \& Okabe, S. 1972, Nucl. Instrum. Methods Phys. Res., 103, 85

Tielens, A. G. G. M. 1998, ApJ, 499, 267

Welty, D. E., et al. 1999, ApJ, 512, 636

Werner, M., et al. 2004, ApJS, 154, 1

Zubko, V., Dwek, E., \& Arendt, R. G. 2004, ApJS, 152, 211 